\title{
Development and Implementation of a Generic Analysis Template for Structural-Thermal-Optical-Performance Modeling
}

\author{
Salvatore Scola, Rebecca Stavely, Trevor Jackson, Charlie Boyer, \\ Jim Osmundsen, Craig Turczynski, Chad Stimson \\ NASA Langley Research Center, 100 NASA Drive, Hampton, VA, USA 23681
}

\begin{abstract}
Performance-related effects of system level temperature changes can be a key consideration in the design of many types of optical instruments. This is especially true for space-based imagers, which may require complex thermal control systems to maintain alignment of the optical components. Structural-Thermal-Optical-Performance (STOP) analysis is a multi-disciplinary process that can be used to assess the performance of these optical systems when subjected to the expected design environment. This type of analysis can be very time consuming, which makes it difficult to use as a trade study tool early in the project life cycle. In many cases, only one or two iterations can be performed over the course of a project. This limits the design space to best practices since it may be too difficult, or take too long, to test new concepts analytically. In order to overcome this challenge, automation, and a standard procedure for performing these studies is essential. A methodology was developed within the framework of the Comet software tool that captures the basic inputs, outputs, and processes used in most STOP analyses. This resulted in a generic, reusable analysis template that can be used for design trades for a variety of optical systems. The template captures much of the upfront setup such as meshing, boundary conditions, data transfer, naming conventions, and post-processing, and therefore saves time for each subsequent project. A description of the methodology and the analysis template is presented, and results are described for a simple telescope optical system.
\end{abstract}

Keywords: structural thermal optical performance analysis, STOP, analysis template, NASA Langley

\section{INTRODUCTION}

Structural-Thermal-Optical-Performance (STOP) analysis is a challenging, yet essential design step for many spaceflight optical instruments and sensors. It is used to verify that an optical system will meet its performance requirements in its expected worst case operating environments. These environments can include effects of varying temperature, vibrational loads, and gravity. A simplified STOP analysis process is shown in Figure 1; first the optical and mechanical systems are defined, followed by the thermal analysis, structural analysis, and optical analysis, in that order. A significant amount of engineering time is spent developing the individual models in each discipline, performing the individual analyses, and translating results from each model into a format that the next model can understand. The cost of performing these analyses generally limits the number of trade studies and configurations that can be assessed during a project.

In most cases, organizations use independent, commercial analysis tools, with customized scripts to perform data transfers between disciplines. This has the advantage of allowing engineers to use software that they are familiar with. A disadvantage to this method is the need to manually convert output from one analysis software into input for the next. There are a number of ways to perform the conversions with the various commercial tools (Bolognese and Irish ${ }^{1}$ ), however there is typically some custom scripting involved. A second disadvantage is the need for each discipline engineer to wait for the results from any upstream analyses that are required. This can cause significant delays if engineers are not available to complete their part of the analysis when it is their turn. A third disadvantage is the potential for human errors to be introduced in each step of the process. Examples of these errors include different material properties specified in different models for the same part, or even differing versions of parts between models. A final disadvantage is that all the models required to compute a final solution are stored in various places at the organization, and there is not a single STOP model than can be packaged and distributed. This makes additional analysis difficult if it is needed in later phases of the project after the STOP team has moved on to other tasks, or other projects.

In order to address the disadvantages inherent in the typical STOP analysis process described above, a generic template for performing STOP analyses was developed within the framework of the commercial software package Comet ${ }^{2}$. The template utilizes CAD-based analysis processes that automate the data transfer between all disciplines. A standard naming 
convention is defined within the template that is applied to the CAD model and to the optics model. This lets the team define the system directly in the CAD model, which allows analyses to be repeated rapidly for any mechanical design change. All loads, boundary conditions, and material properties are managed within the single template which significantly reduces the potential for human errors in the analysis. The template also enables easy packaging and transfer of a complete STOP model that can be re-run anytime in the future, possibly by a single engineer, depending on the complexity of the desired analysis.

The ability to re-use the same template across different projects is also a significant advantage, and a key difference between similar analyses performed using Comet, where a project specific template was used $\left(\mathrm{Geiss}^{3}, \mathrm{Scola}^{4}\right)$. As long as the same, standardized naming conventions are used across projects, STOP analysis for most optical systems can be completed with the same template. As new features are added to the template, they become available for all future projects that may require them.

The purpose of this paper is to describe the template in detail, and discuss the advantages of its use. Example analyses for two completely different optical concepts for the same instrument will be presented, including a discussion of the time required to perform the analyses. It is estimated that the overall time required to perform trade studies and analyses can be reduced by up to $75 \%$ when compared to using independent analysis tools with customized scripting for each project.

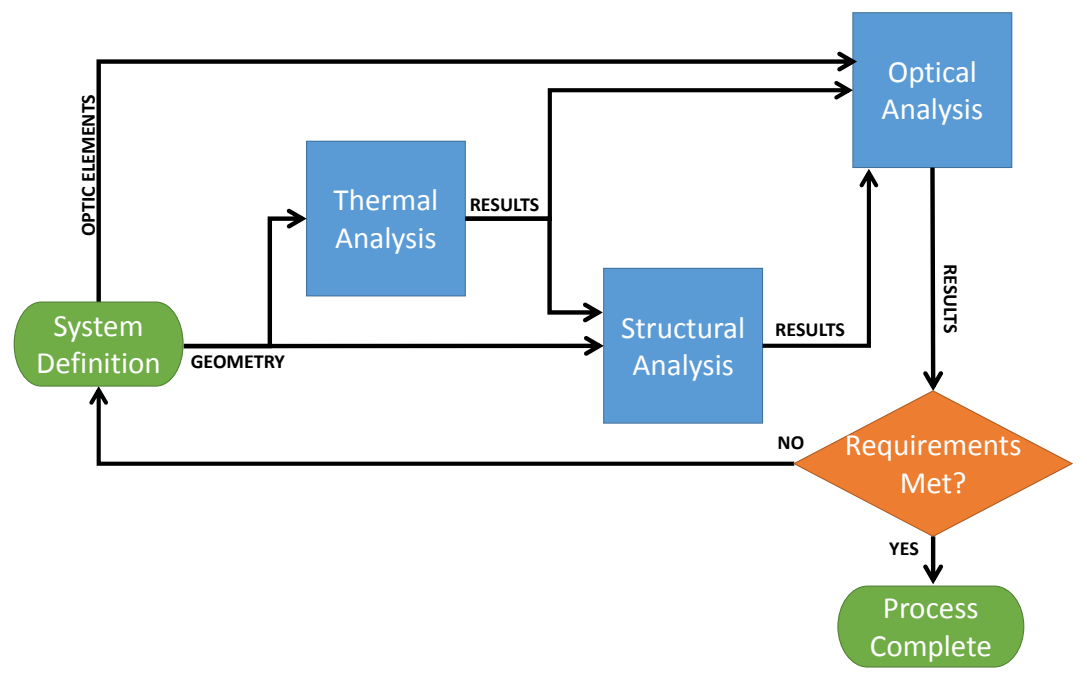

Figure 1. Typical STOP analysis process

\section{GENERIC STOP TEMPLATE DEVELOPMENT AND DESCRIPTION}

The basic components of the generic STOP template are shown in Figure 2. The "STOP Template" in this figure is a Comet project file that serves as the main interface to the model, and is shown in Figure 3. The primary components that allow the generic template to be re-used for most optical systems are the geometric naming convention, the optical naming convention, and a default set of generic processes. The geometric naming convention defines a standard set of names that are used to represent parts (optical components, mounts, optical benches), assemblies (such as the system to be meshed and analyzed), and a number of other features such as surfaces, edges, points, and coordinate systems. This naming convention tells the template how to mesh the system, what material properties to apply, and how loads and boundary conditions should be handled. The optical naming convention is a standard way to name each surface in the optical model, and relate these surfaces to their representations in the CAD model.

The basic procedure for performing an analysis with the template is shown in Figure 4. The first steps are to define the requirements that need to be verified with the STOP analysis, set a common unit system, choose a global coordinate system, and specify material properties. Next, an optics model is developed using Code VTM, and the surfaces of this 
model are named following the standard naming convention. A mechanical design is then developed to support the optics, and a simplified CAD representation of the system is created using CREO ${ }^{\circledR}$. The CAD model should be developed for the sole purpose of analysis, and should be simplified to allow a mesh to be created easily. The mechanical, structural, and thermal engineers work closely on this step to ensure the geometry is properly simplified for the analysis. After the CAD model is complete, the generic template is customized for the specific design to be analyzed. This includes setting default material properties on all the parts, setting up default loads and boundary conditions, modifying variables that control process inputs and requirements, adding new components to the naming convention, and adding or removing features from specific processes. It should be noted that no CAD or optics models have yet been imported into the template, and all these changes are made generically based on the naming conventions. The next step is to apply the geometric and optical naming conventions to the $\mathrm{CAD}$ model using a $\mathrm{CREO}^{\circledR}$ plugin provided with the Comet software in a process called tagging. After tagging, the CAD and optics models can be imported into the generic template. Once imported, meshing tests and sensitivity studies can be performed to find the desired meshing parameters for the system. The final step is to run through the trade studies that need to be performed. A more detailed description of this process will be provided in the following subsections.

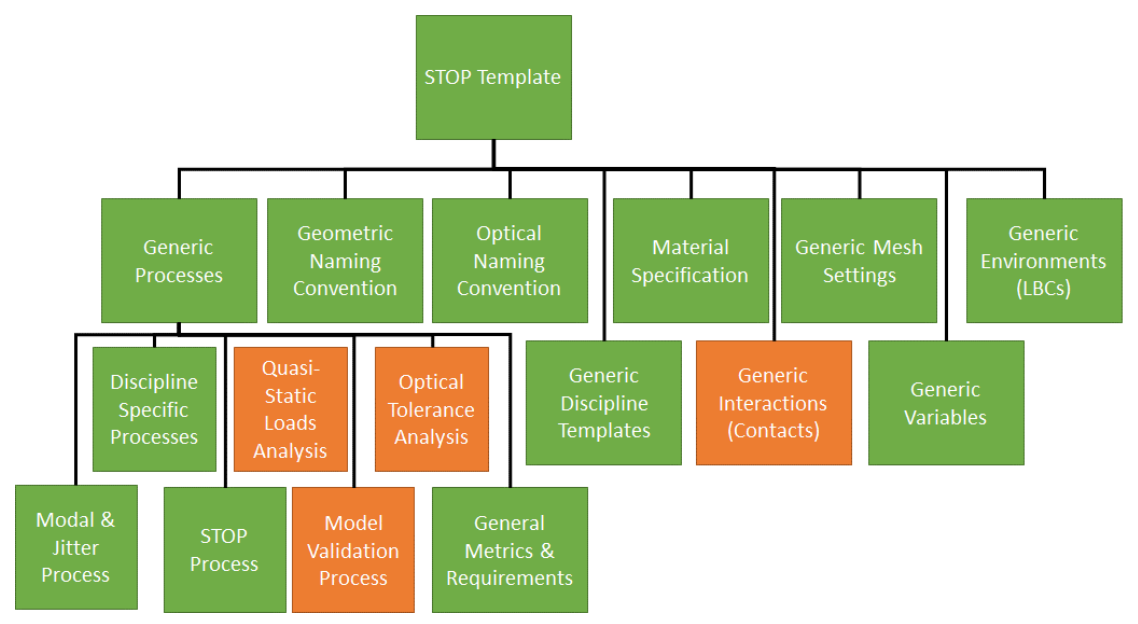

Figure 2. Components of the generic template. Green: completed components. Orange: in development

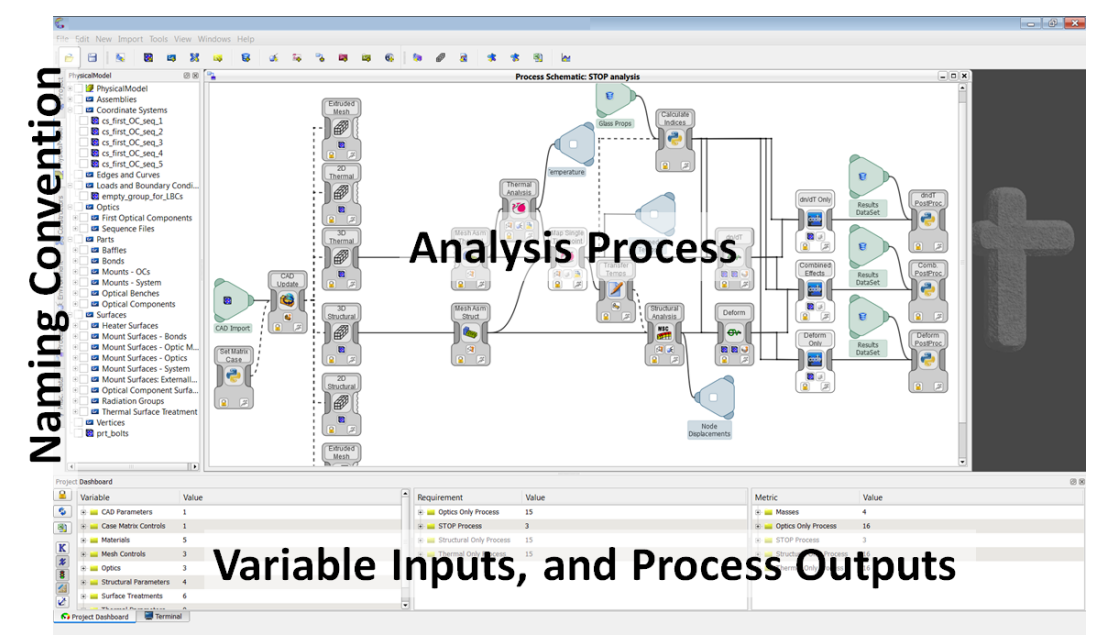

Figure 3. Generic template Comet project 


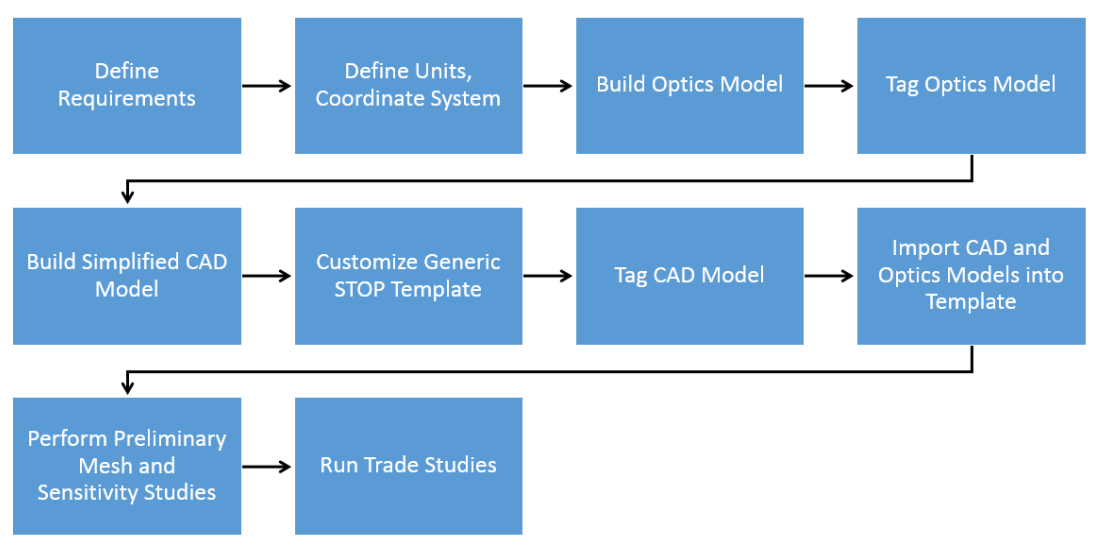

Figure 4. Procedure for performing an analysis with the template

\subsection{Geometric and Optical Naming Conventions}

The naming convention developed for the template is the primary link between the analyses and the physical systems. The fact that optical systems share similar functions and have similar components allows the same naming convention to be used across all designs, which is one of the primary benefits of the template. CAD geometry for a particular system is tagged using these conventions, which are pre-defined in the template. Once all of the parts and surfaces are assigned names in the CAD environment, then the geometry will automatically populate into the correct groups when the CAD is imported into Comet. Geometry needs to be tagged in order for Comet to manipulate it.

Table 1 through Table 3 list some examples of the geometric naming convention that was developed. All parts are named with the prefix "prt_" and the numbering of all parts follows that of the closest-associated optical component (Table 1). The first optical component in the optical path is numbered 101 and the rest are numbered sequentially along the optical path. For the first optical component in the path "prt_OC_101", the mounting structure would be called "prt_mount_OC_101" and the optical bench it is fixed to would be called prt_OB_101.

Multiple geometric parts can be collected under a single name if required. For instance, if a lens is mounted using a 3point-bond system then all three bonds can be grouped together under a single part name rather than naming each bond individually.

All surfaces to be used in the analysis are named with the prefix "surf_" (Table 2). Not all surfaces need to be specifically named, but a surface needs to be specifically tagged in order to do the following:

- Indicate that a surface is active in radiation thermal transfer analysis

- Apply a boundary condition or load to a particular surface

- Apply unique radiation properties (emissivity and solar absorptivity) to a particular surface of a part that is different from the rest of the part

All assemblies to be used in the analysis are named with the prefix "asm_" (Table 3). These groups are used to define mesh domains for the analysis. The asm_2D assembly group collects all of the two-dimensional parts so that the mesh metrics can be controlled separately from the three-dimensional parts which are stored in the asm_3D group. The asm_thermal_analysis_mesh and asm_structural_analysis_mesh groups represent the meshes that are passed to the thermal and structural analysis tasks, and can include a combination of 2D and 3D meshes. The parts associated with these assembly groups are manually updated in the Comet workspace for each project. 
Table 1. Some examples of the geometric naming convention for parts used in the analyses

\begin{tabular}{|l|l|l|}
\hline Item & Naming Convention & Descriptions \\
\hline \multirow{5}{*}{ Parts } & prt_OC_\#\#\# & $\begin{array}{l}\text { Each optical component }(\mathrm{OC}) \text { named in order from the first component in the } \\
\text { optical path }(101,102, \ldots)\end{array}$ \\
\cline { 2 - 3 } & prt_mount_OC_\#\#\# & The mounting apparatus of each optical component $(101,102, \ldots)$ \\
\cline { 2 - 3 } & prt_bond_OC_\#\#\# & The bonds between an optical component and its mounting fixture $(101,102, \ldots)$ \\
\cline { 2 - 3 } & prt_OB_\#\#\# & $\begin{array}{l}\text { The optical bench to which the optical component mounts are attached } \\
(101,102, \ldots)\end{array}$ \\
\cline { 2 - 3 } & prt_OC_Image & Imaging optical component, detector \\
\cline { 2 - 3 } & prt_ & General prefix for adding additional parts \\
\hline
\end{tabular}

Table 2. Some examples of the geometric naming convention for surfaces used in the analyses

\begin{tabular}{|c|c|c|}
\hline Item & Naming Convention & Descriptions \\
\hline \multirow{6}{*}{ Surfaces } & surf_ext_rad_group_\# & $\begin{array}{l}\text { Assigning surfaces to be part of radiation transfer in thermal analysis. "ext" } \\
\text { stands for an "external" group to help differentiate groups as for the space- } \\
\text { facing surfaces in orbit analysis }(1,2, \ldots)\end{array}$ \\
\hline & surf_int_rad_group_\# & $\begin{array}{l}\text { Assigning surfaces to be part of radiation transfer in thermal analysis. "int" } \\
\text { stands for an "internal" group to help differentiate groups as for surfaces } \\
\text { which exchange radiation with the interior of the optics system enclosure } \\
(1,2, \ldots)\end{array}$ \\
\hline & surf_Heater_\# & Assigning a surface where a heater may be applied $(1,2, \ldots)$ \\
\hline & surf_mount_sys_\#\#\# & $\begin{array}{l}\text { Surfaces for boundary conditions where each optical bench attaches to } \\
\text { surrounding structure, such as inside of a satellite, and may have boundary } \\
\text { conditions applied }(101,102, \ldots)\end{array}$ \\
\hline & surf_ST_ & $\begin{array}{l}\text { Name and assign a surface with unique radiation properties (surface } \\
\text { treatment, "ST"). Use this when one surface of a part has a different } \\
\text { coating that the other part's surfaces. }\end{array}$ \\
\hline & surf & General prefix for adding additional parts \\
\hline
\end{tabular}

Table 3. Some examples of the geometric naming convention for assemblies used in the analyses

\begin{tabular}{|l|l|l|}
\hline Item & Naming Convention & Descriptions \\
\hline \multirow{4}{*}{ Assemblies } & asm_CAD_UPDATE & $\begin{array}{l}\text { Captures all geometry and coordinate systems to be imported into } \\
\text { Comet. }\end{array}$ \\
\cline { 2 - 3 } & asm_2D & 2D mesh assembly, manually updated in Comet per design \\
\cline { 2 - 3 } & asm_3D & 3D mesh assembly, manually updated in Comet per design \\
\cline { 2 - 3 } & asm_struc_analysis_mesh & Structural mesh component, manually updated in Comet per design \\
\cline { 2 - 3 } & asm_therm_analysis_mesh & Thermal mesh component, manually updated in Comet per design \\
\cline { 2 - 3 } & asm_ & General prefix for adding additional assemblies \\
\hline
\end{tabular}


Table 4 describes the optical system naming convention, which is used in the optics model and in the CAD model. In the optics model, each surface that will be part of the analysis is named using the convention on the first row of Table 4 . The associated surface in the CAD model is also tagged with the same name so the analysis process can link the deformations of a particular mesh to the optics model. The coordinate system of the first optical component in the active optical path must be tagged in the CAD model so that the optics model can be properly aligned with the CAD model.

Figure 5 shows an example of the naming convention applied to an optical component. Figure 6 shows how the naming conventions are stored within the Comet template as groups. These groups collect associated objects from the CAD and optics models when they are imported, as shown in Figure 7. Each geometry group has a material property and radiation property (surface treatment) which are applied to all parts imported into the group. Surfaces which are tagged to specify a surface treatment name, for example surf_ST_Mirror, are automatically populated into the surf_ST_Mirror group which will override the surface treatment that was applied globally to the associated part.

Table 4. Some examples of the optical naming convention used in the analyses

\begin{tabular}{|c|c|c|}
\hline Item & Naming Convention & Descriptions \\
\hline \multirow[t]{3}{*}{$\begin{array}{l}\text { Optical } \\
\text { Components }\end{array}$} & OC_\#\#\#_OS_\# & $\begin{array}{l}\text { Each surface in the optical system is named with the same names as are } \\
\text { used in the Code } V^{\mathrm{TM}} \text { sequence file. Optical component }(\mathrm{OC} 101,102, \ldots) \\
\text { and optical surface (OS } 1 \text { or } 2 \text { ). First optical component surface in the } \\
\text { path is called OC_101, and the first optical surface on each component is } \\
\text { OS_1. Lenses have two unique surfaces ('in' and 'out') while mirrors only } \\
\text { have one surface. }\end{array}$ \\
\hline & OC_Image & Surface tag for where the final image is taken \\
\hline & Cs_first_OC_seq_\# & $\begin{array}{l}\text { Code } V \text { needs an input for the coordinate system of where the first } \\
\text { optical component in the optical sequence is located. \# for each unique } \\
\text { optical path }\end{array}$ \\
\hline
\end{tabular}

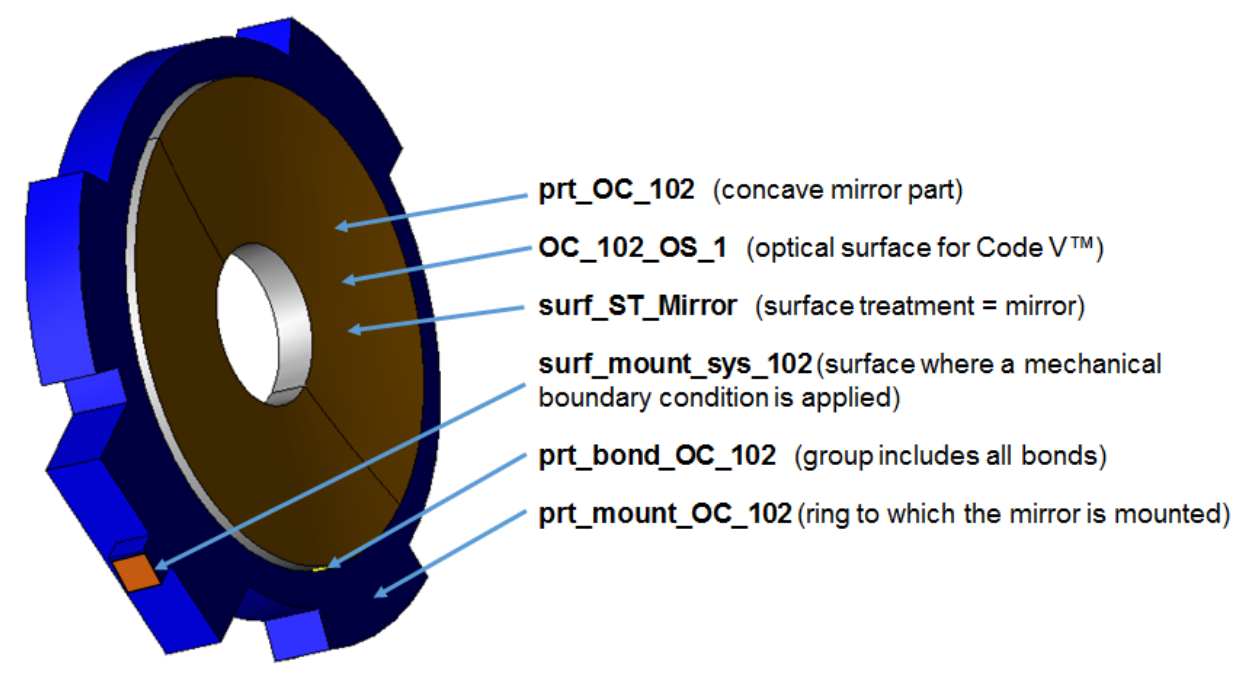

Figure 5. Example of naming convention for parts and surfaces around Optical Component 102 

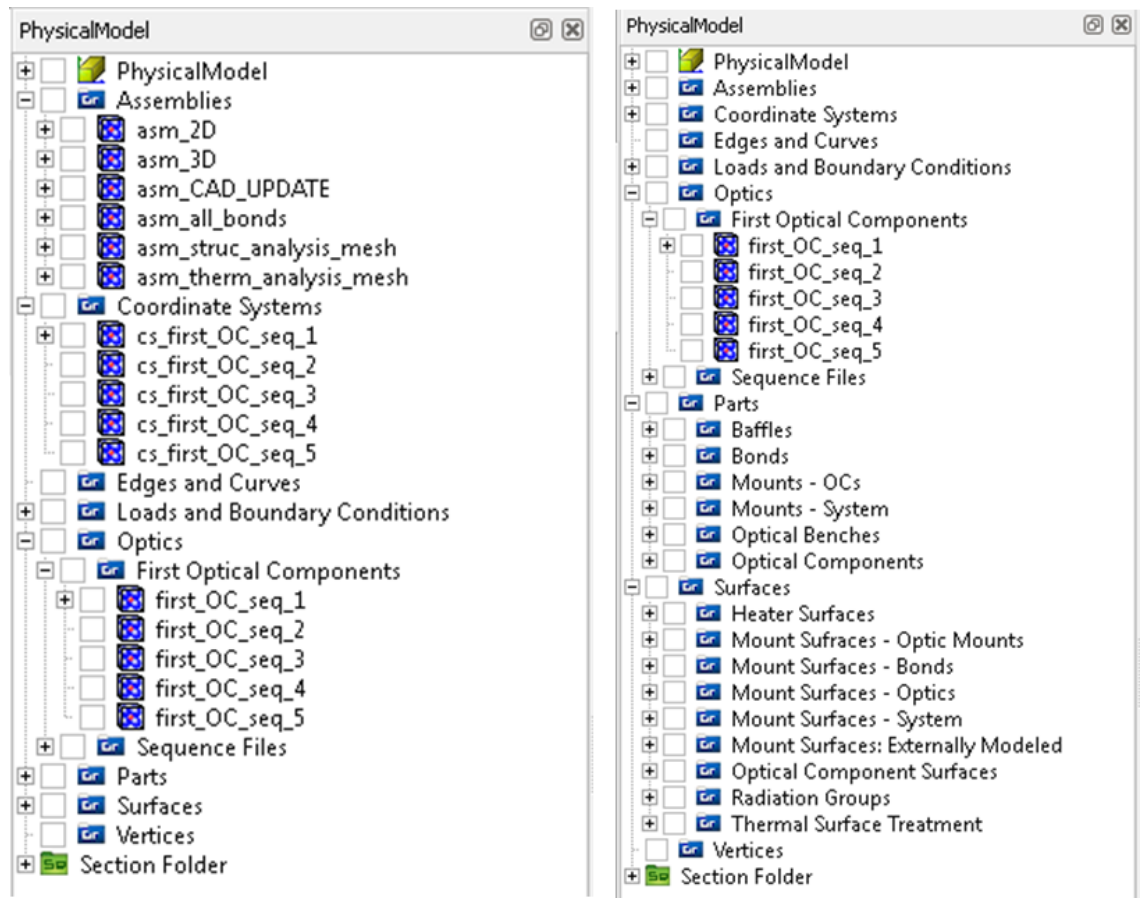

Figure 6. Template of empty groups which automatically becomes populated when the tagged CAD model is imported

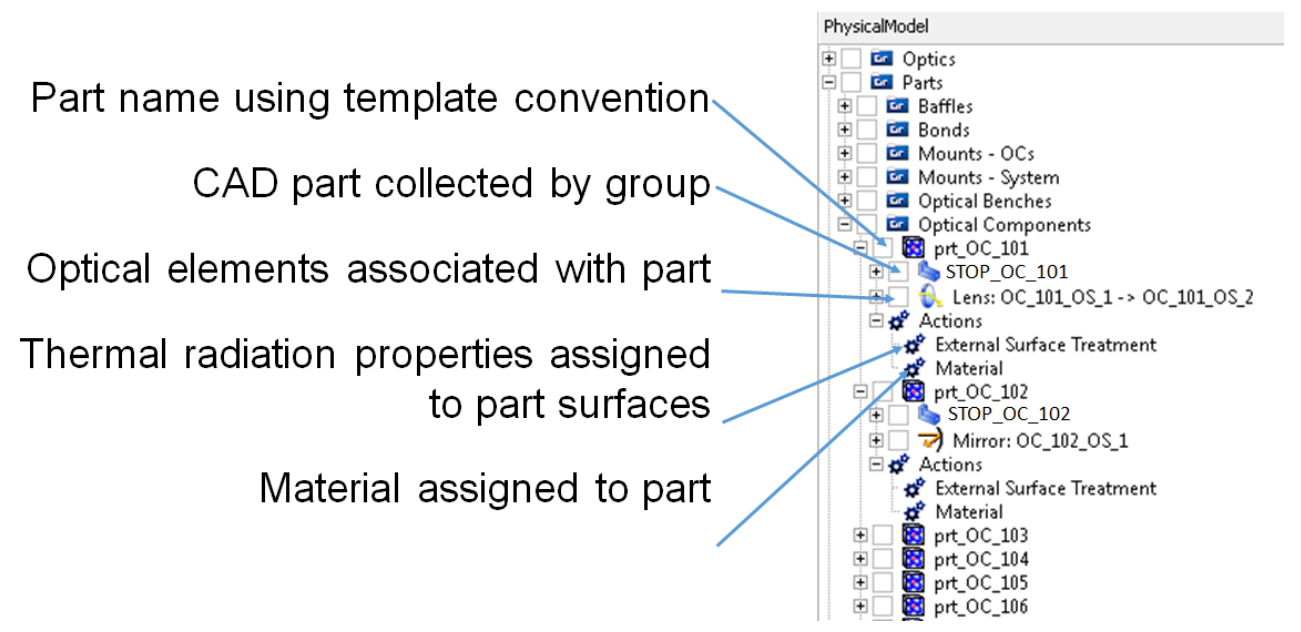

Figure 7. Example of template with CAD and Optics included, showing actions that apply material properties.

\subsection{Material Properties, Loads and Boundary Conditions}

Solid material properties are applied to parts using variables within Comet, and an external material library file. The naming convention of these material variables is similar to the geometry tags, and are described in Table 5. The material and surface treatment actions shown in Figure 7 are linked to these variables. A list of available properties is kept up to date and can be copied and pasted into the variable values for each part, as shown in Figure 8. When material properties 
and thermal surface properties are applied to a part, the application of the properties is global. If one surface of the part requires a different type of surface treatment it can be applied specifically to that surface only.

Table 5. Variable names for material properties.

\begin{tabular}{|c|l|l|}
\hline \multirow{2}{*}{ Item } & $\begin{array}{l}\text { Variable Naming } \\
\text { Convention }\end{array}$ & Descriptions \\
\hline \multirow{3}{*}{$\begin{array}{c}\text { Material } \\
\text { Properties }\end{array}$} & Mat_OC_\#\#\# & Material designation for each optical component $(101,102, \ldots)$ \\
\cline { 2 - 3 } & Mat_OB_\#\#\# & Material designation for each optical bench $(1,2, \ldots)$ \\
\cline { 2 - 3 } & Mat_mount_OC_\#\#\# & Material designation for each optical component mount $(101,102, \ldots)$ \\
\hline \multirow{2}{*}{$\begin{array}{c}\text { Surface } \\
\text { Treatments }\end{array}$} & ST_OC_\#\#\# & Surface treatment for all surface of an optical component $(101,102, \ldots)$ \\
\cline { 2 - 3 } & ST_ & $\begin{array}{l}\text { General designation of any surface treatment, especially unique names for } \\
\text { special surfaces such as applying MLI to just one side of a part }\end{array}$ \\
\hline
\end{tabular}

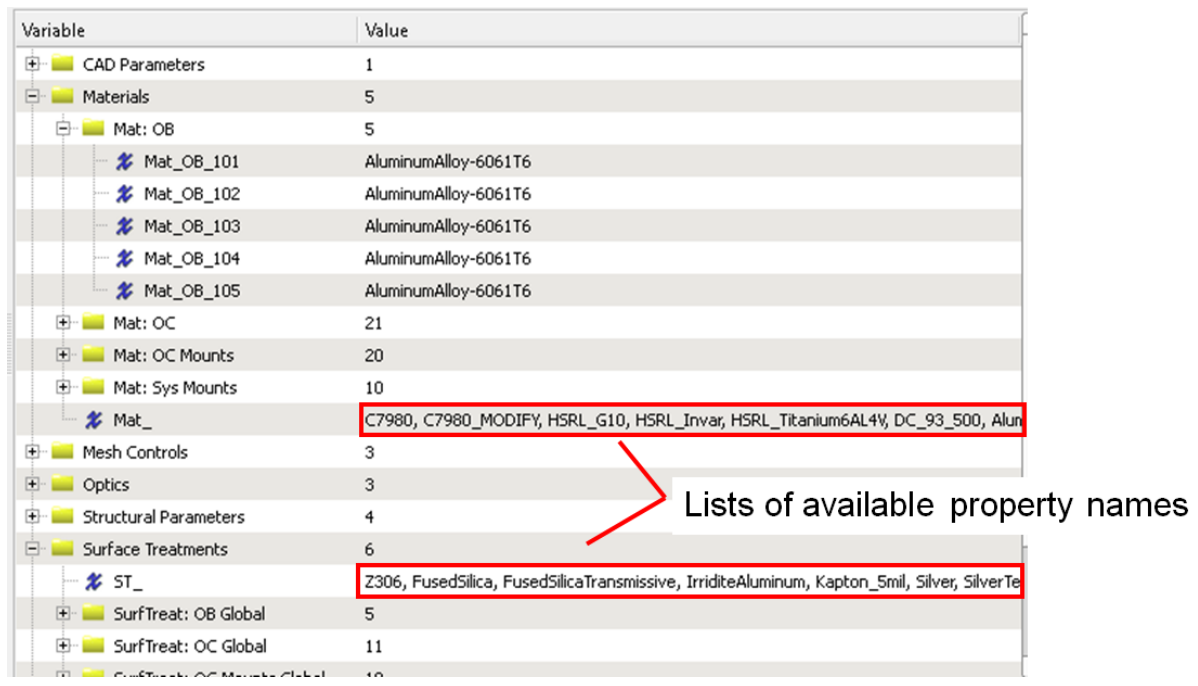

Figure 8. Material and surface treatment variables, linked to part and surface groups.

Boundary conditions can also be applied to specific surfaces that have been tagged in CREO ${ }^{\circledR}$. The part groups can also be used to apply global conditions like initial temperatures. Many of the common loads and boundary conditions such as initial temperature and fixed mechanical boundary conditions have been connected in the template and only require geometry to be tagged in order to begin performing analyses and trade studies from the dashboard. Applying loads and boundary conditions specific to a particular project is a manual task for each project; a new load or boundary condition is created and then linked to a desired part or surface group.

Some loads are pre-made in the template and only require a surface to be tagged in order to activate the load. For example, many of the parameters for heaters have already been created in the template to be controlled via the dashboard and only require that some geometry be appropriately tagged in the CAD model. An example of these heaters are shown in Figure 9. 


\begin{tabular}{|c|c|c|c|c|c|}
\hline Variable & \multirow{2}{*}{$\begin{array}{l}\text { Value } \\
9\end{array}$} & & & & \\
\hline$\square-$ Thermal Parameters & & & & & \\
\hline+ Analysis Case Options & 6 & SensorDomain & ApplicationDomain & Properties & Value \\
\hline Heater Settings & 20 & $\square$ surf_Heater_1 & $\square$ surf_Heater_1 & - off Temperature & $20.5{ }^{\circ} \mathrm{C}$ \\
\hline \# H1_Proportional & true & & & onTemperature & $19.5^{\circ} \mathrm{C}$ \\
\hline$-\boldsymbol{x} \mathrm{H}_{1} \_\mathrm{Q}$ & $10 \mathrm{~W}$ & & & (t) powerSettings & HeaterPowerSettings \\
\hline * H1_T_DeadBand & $1{ }^{\circ} \mathrm{C}$ & & & senseMethod & AREA WEIGHTED AVERAGE TEMPERATURE \\
\hline * H1_T_Setpoint & $20^{\circ} \mathrm{C}$ & & & † steadyStateBehavior & \\
\hline - $\boldsymbol{*}$ H2_proportional & true & & & & \\
\hline$-x \mathrm{H}_{2} \mathrm{QQ}$ & $10 \mathrm{~W}$ & & & & \\
\hline
\end{tabular}

Figure 9. Heater settings for pre-defined heater surfaces included in the template.

\subsection{Analysis Processes and Requirements Verification}

The template currently includes several analysis processes that can be used to assess the optical performance of the system. As an example, the full STOP process is shown in Figure 10. This process includes the ability to perform a full thermal analysis using Thermal Desktop, map the temperature results to a NASTRAN structural model, and compute both dn/dT and deformation effects on performance with SigFit and Code V. Each blue rectangular box represents a specific analysis task, and the lines between boxes represent data being passed from one task to the next. The process starts with the definition of the CAD assembly to be meshed. The user determines which parts to use in the analysis by modifying the parts that are associated with the assembly group passed to the mesh tasks. By default, the group that is passed to the mesher includes all parts in the geometric naming convention.

Determining what mesh parameters work best for the particular geometry being analyzed is one of the more manual steps when performing an analysis. It can take several iterations to get a mesh that is acceptable for both thermal and structural analyses. Once the mesh is determined however, the same mesh can be used for all trade studies. The size settings for the mesh are controlled with Comet variables which are linked to all meshing tasks, in all processes in the template, so the user only needs to set them once.

The thermal analysis can also take a few iterations during the initial setup of a new design. The thermal task uses a template Thermal Desktop file, that can contain other parts of the system that are not being directly analyzed in the STOP analysis; for example, the spacecraft bus. Ensuring that the translation of the FEM model from Comet into the Thermal Desktop file is correct is one of the required steps in the process. Once these manual steps have been performed, the settings are saved, and all future trade studies can be run automatically.

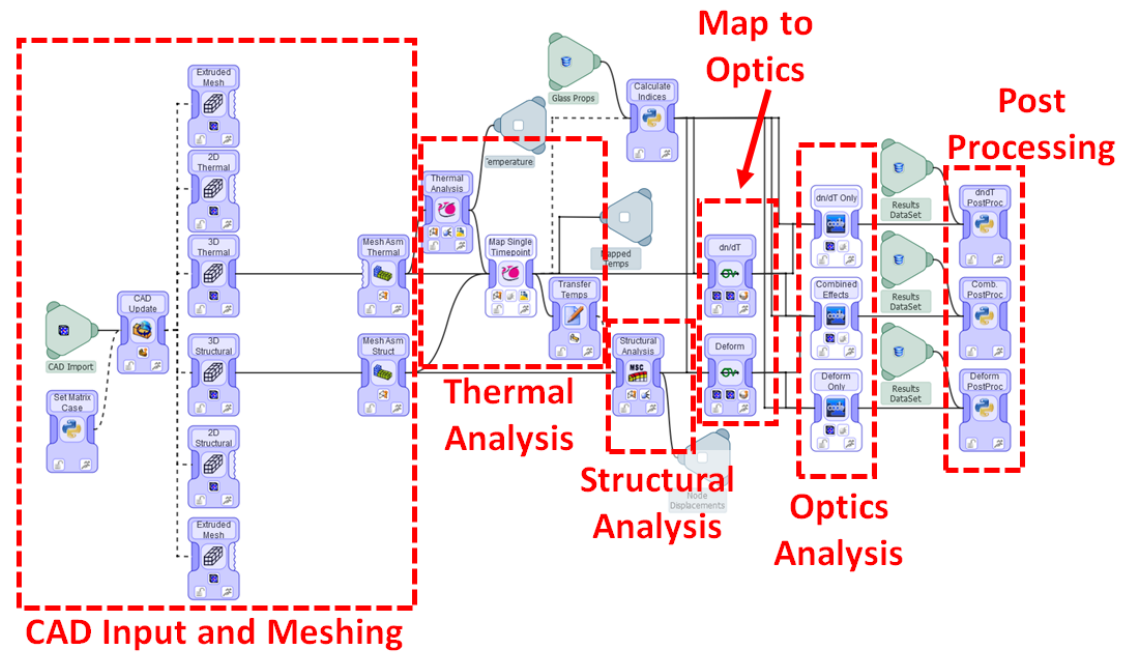

Figure 10. Full STOP process. 
Each process in the template is linked to a set of optical performance metrics that can be used to verify whether the system meets its requirements for each analysis performed. The template is currently set up to evaluate defocus, modulation transfer function (MTF) at a user specified spatial frequency, and encircled energy for a user specified spot diameter. A metric is also included that calculates the mass of the assembly being analyzed, and allows a quick mass comparison between material and geometric trade studies. Figure 11 shows an example of the output in the Comet template. At the completion of an analysis, individual items in the list will turn green if the requirement is met, or red if it is not met. This information is provided for all field points (FP) in the optics model. In this particular case, the encircled energy is being evaluated at $15 \mu \mathrm{m}$ diameter, and the MTF is being evaluated at $100 \mathrm{lp} / \mathrm{mm}$. Not shown is a set of constraints that defines the green (passing) limits for each requirement.

Each analysis task in the process includes the ability to customize the input and/or output files of the analysis program specific to the task. This customization is achieved with Python scripting, and can be a very useful way to include customizations that have been developed on other programs into the analysis. The template uses such scripts in several places. As an example, a script is used to ensure the refractive index and dn/dT for each glass being used is calculated appropriately based on the selected wavelength, initial temperature, and final temperatures used in the analysis process. It also ensures SigFit and CodeV use the same values for these properties.

\begin{tabular}{|c|c|c|c|}
\hline Variable & Value & Requirement & Value \\
\hline † CAD Parameters & 1 & Structural Only Process & 16 \\
\hline+ Case Matrix Controls & 1 & + \$ struconly_EncEngy_FP1 & 0.9375 \\
\hline$+\backsim$ Materials & 7 & + \& struconly_EncEngy_FP2 & 0.9375 \\
\hline+- Mesh Controls & 3 & + ३ै struconly_EncEngy_FP3 & 0.9375 \\
\hline$=$ Optics & 3 & + \& struconly_EncEngy_FP4 & 0.925 \\
\hline * Encircled_Energy_Selected_Diameter & $15 \mu \mathrm{m}$ & + \& struconly_EncEngy_FP5 & 0.95 \\
\hline * MTF_Selected_Frequency & $1001 / \mathrm{mm}$ & + \$ struconly_defocus & $-0.05596 \mathrm{~mm}$ \\
\hline $\boldsymbol{*}$ Wavelength & $350 \mathrm{~nm}$ & + \& struconly_XMTF_FP1 & 0.273666666666666 \\
\hline+ Structural Parameters & 4 & + हैं struconly_XMTF_FP2 & 0.268444444444444 \\
\hline+- Surface Treatments & 6 & + हैं struconly_XMTF_FP3 & 0.2865555555555555 \\
\hline \multirow[t]{2}{*}{ †. Thermal Parameters } & 9 & + हैं struconly_XMTF_FP4 & 0.353444444444444 \\
\hline & & + \# struconly_XMTF_FP5 & 0.514555555555555 \\
\hline
\end{tabular}

Figure 11. Comet template requirements. The left pane shows the user selected values that are used to verify the requirements. The right pane indicates whether a requirement is met (green) or not met (red) for a particular analysis case.

In addition to the full STOP process described above, the template also includes:

- An optics-only process which allows trade studies to be performed on the optics model alone.

- A uniform temperature change model, in which the thermal model is replaced with a uniform temperature load on the structural model. This significantly increases the speed of the analyses, and is very useful for preliminary design.

- A thermal-only process, which allows testing of the thermal model, and running dn/dT only simulations

- A normal modes and jitter/line of sight process which calculates the normal modes of the system, and uses this to compute the effect on performance when subjected to a vibrational disturbance on-orbit.

- A set of processes that allow a case matrix of analyses to be run using one of the above-listed analyses process. A user can enter the different combinations of variables defining each case in an Excel spreadsheet. This allows a large set of analyses to be run in an automated fashion.

\section{DESIGN STUDY FOR A SIMPLE IMAGING SYSTEM}

The STOP template was used to compare two very different optical systems designed to meet the same set of requirements. The primary mission requirement is to capture greater than $50 \%$ of the point spread function encircled energy within a $15 \mu \mathrm{m}$ diameter, for the field points shown in Table 6 . This diameter translates to about half the size of a single pixel on the selected detector. The requirement applies to an input wavelength of $1100 \mathrm{~nm}$, however the encircled energy should 
also be reported at $350 \mathrm{~nm}$ and $587 \mathrm{~nm}$. Figure 12 shows the optical and mechanical designs for each of the two configurations studied. The first case is a Ritchey-Chrétien design, consisting of a primary and secondary mirror, and the second case is an off-axis design with 8 mirrors. The off-axis design is more complex than the Ritchey-Chrétien design, but it has the advantage of higher baseline encircled energy due to the lack of the central obscuration present in the RitcheyChrétien. As shown in the figure, the Ritchey-Chrétien design is $10 \%$ above the $50 \%$ requirement at $1100 \mathrm{~nm}$ for field points 3 and 5 . The off-axis design is about $25 \%$ higher at $1100 \mathrm{~nm}$, and more uniform across all field points. Both of these plots were generated using the Optics Only process in the STOP template.

The optical system will be flown on a power-limited spacecraft, where thermal control may not be possible. The environment temperature surrounding the optical system could therefore range from $-30^{\circ} \mathrm{C}$ to $+60^{\circ} \mathrm{C}$. It is also desired to keep the first mode of the system above $50 \mathrm{~Hz}$. On-orbit disturbances were unknown at the time of the analysis, so jitter and line of sight analyses were not performed. The initial temperature of the system is assumed to be $20^{\circ} \mathrm{C}$ in all analyses.

The following subsections outline the analyses that were performed with the STOP template to compare the two designs. Even though the two systems are very different, the same template can be used since the analyses, and requirements required for each are the same. All that is required is to apply the same geometric and optical naming conventions to each CAD model. This is the primary benefit of using a common template, and it can save a significant amount of repeated setup work across designs.

Table 6. Analysis field points

\begin{tabular}{|l|l|l|}
\hline Field Point & X Angle & Y Angle \\
\hline FP1 & 0.0 & 0.0 \\
\hline FP2 & 0.0 & -0.5 \\
\hline FP3 & 0.0 & 0.5 \\
\hline FP4 & -0.5 & 0.0 \\
\hline FP5 & 0.5 & 0.0 \\
\hline
\end{tabular}



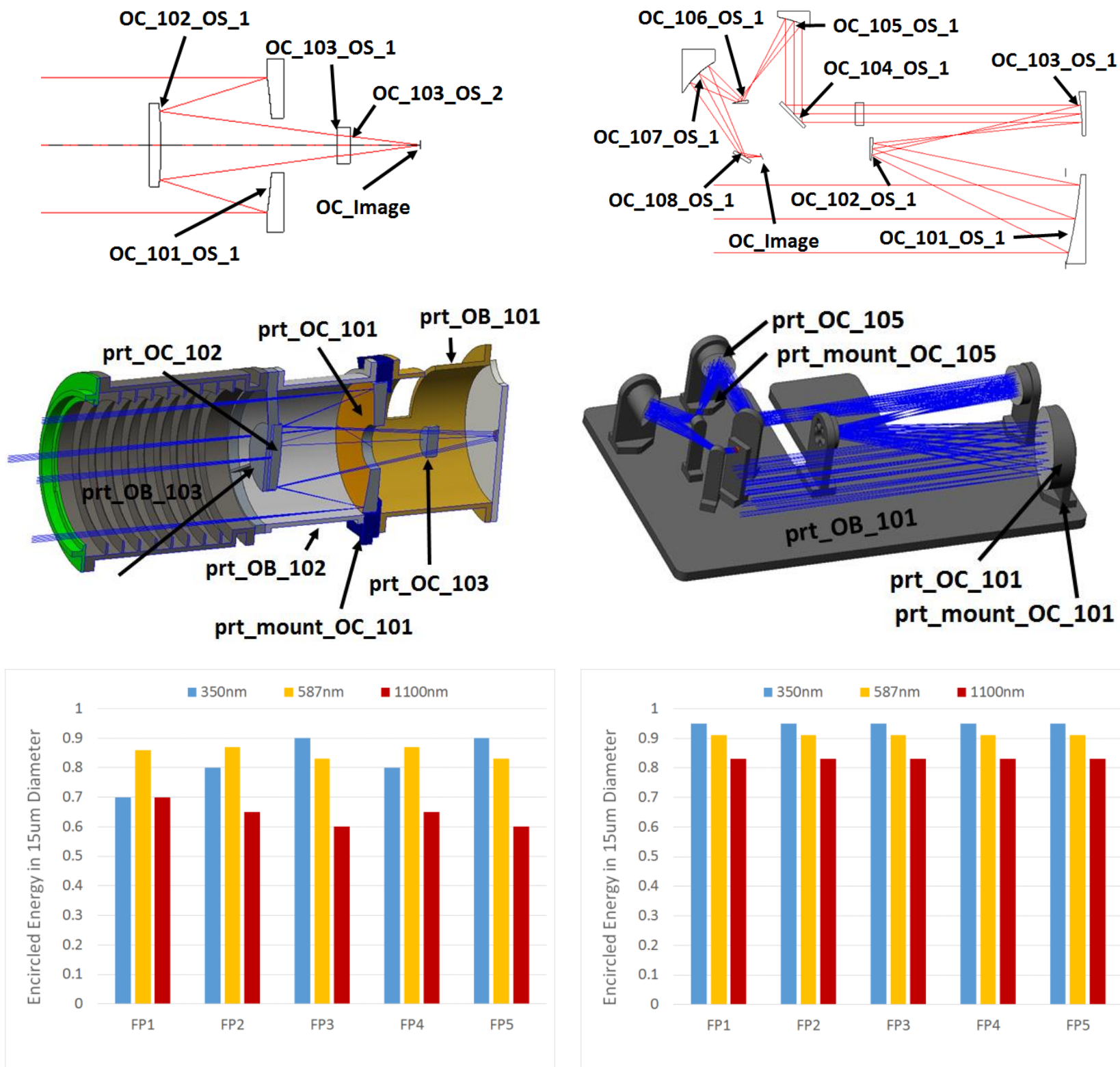

a.) Ritchey-Chrétien design

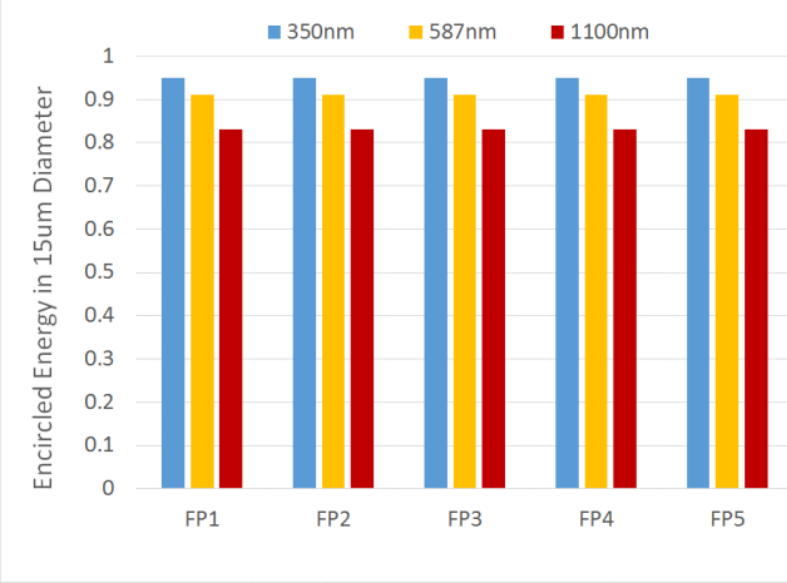

b.) Off-axis design

Figure 12. Two optical configurations studied to meet a common requirement set, shown with a subset of the naming convention applied.

\subsection{Simplified CAD Model Development}

The first step in the analysis process is to build a simplified CAD model for system. This can be the most time intensive part of the process, as it requires input from all the disciplines. The primary goal in this step is to develop a CAD model that easily supports the analyses that need to be performed. This is very different than the typical approach, in which each discipline generates a mesh from a detailed CAD model.

To build a mechanical model, the optical surfaces and placement data for each element is transferred from the optical design software (CODE V) to the mechanical modeling CAD software (CREO 2.0). The sag equation is used for spherical, conoidal, and higher order aspheric surfaces while modeling lenses and mirrors as solid CAD objects. Non-rotationally 
symmetric freeform surfaces based on equations can also be used. These solid CAD model objects are the basis for the optomechanical CAD design work to mount the optical elements. The coordinate system of the CAD model coincides with the optical design coordinate system for consistency. The optical design software provides offset coordinates for each optical element placement from a global zero, which is the basis for creating the CAD model assembly. This ensures cohesion between the optical design and mechanical design.

CAD model assemblies must be simplified to allow meshing to be performed easily, and to provide appropriate areas for application of loads and boundary conditions. This includes removal of detailed parts and features such as rounds, chamfers, and holes not affecting the optical elements. The detailed parts are replaced by simplified parts which maintain the structural and thermal properties of the model, as shown in Figure 13. The level of fidelity can range from simple surfaces to detailed solid parts, or a mix of the two as shown in Figure 14. Geometric dimensions in the CAD model can be parameterized, and driven by the STOP template to perform optimization analyses.

Once the CAD model is completed, the next step is tagging the model with the naming convention used in the template. The naming convention is imported into CREO directly from the Comet template. The user can then go through the list of names, and apply them to specific parts, surfaces, or assemblies in the model. This process generally takes no more than 1 hour to complete, and is the primary way to define how the template and associated analysis processes will manage the parts.
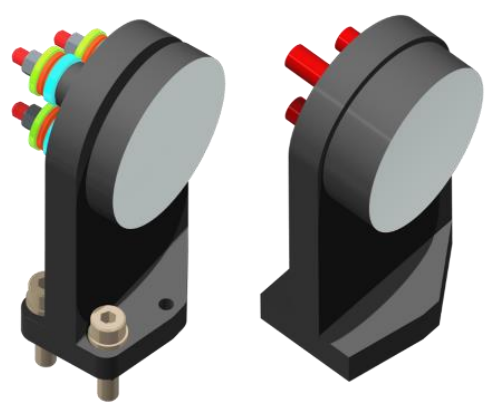

Figure 13. Adjustable mirror design (left). Simplified representation for analysis (right)

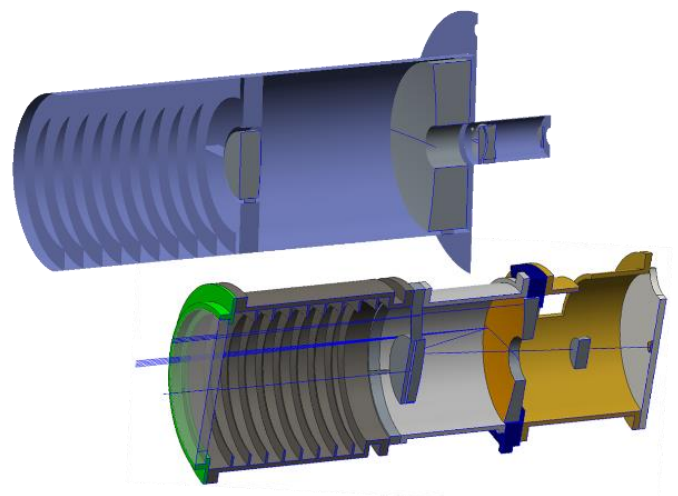

Figure 14. Simple surface model (top) and detailed solid part model (bottom), both used for analysis

\subsection{Material trade study analysis results}

For both designs, a simple material trade study was first performed. Aluminum, invar, and titanium were all assessed as potential materials to build the optical bench, and optical mounts for the system. The off-axis design also considered Silicon Carbide as a potential optical bench construction material. For the off-axis case, silicon carbide is assumed for the mirrors, and the mirror mounts in all cases. Figure 15 compares the results for these studies, and shows that the off-axis design performs better over a wider range of temperatures, for all material choices. This is primarily attributed to the higher performance at room temperature due to the lack of central obscuration. An unexpected result occurs between the titanium and invar optical bench cases for the off axis design; the titanium bench results in a nearly constant encircled energy, and performs better than the invar bench, even though it has a higher coefficient of thermal expansion (CTE). Closer inspection of the analysis results shows that this is due to the different level of CTE mismatch between the optical bench and the silicon carbide optical mounts. In the titanium case, the bending direction of the mounts compensates for the expansion of the bench, while the opposite is true in the invar case. The titanium bench offers even better performance than the silicon carbide bench due to the compensating effect of the mount bending. More investigation is required to determine if this is simply an artifact of the analysis assumptions, or if it is a real effect.

A total of 27 STOP analyses were performed to generate the Ritchey-Chrétien plots in Figure 15. A few additional temperature points were added to the off-axis design plots, for a total of 33 STOP analyses for this design. Using the template, three engineers performed the analyses for the Ritchey-Chrétien design during a daylong training session. The off-axis analyses were performed by a single engineer over the course of one day. 


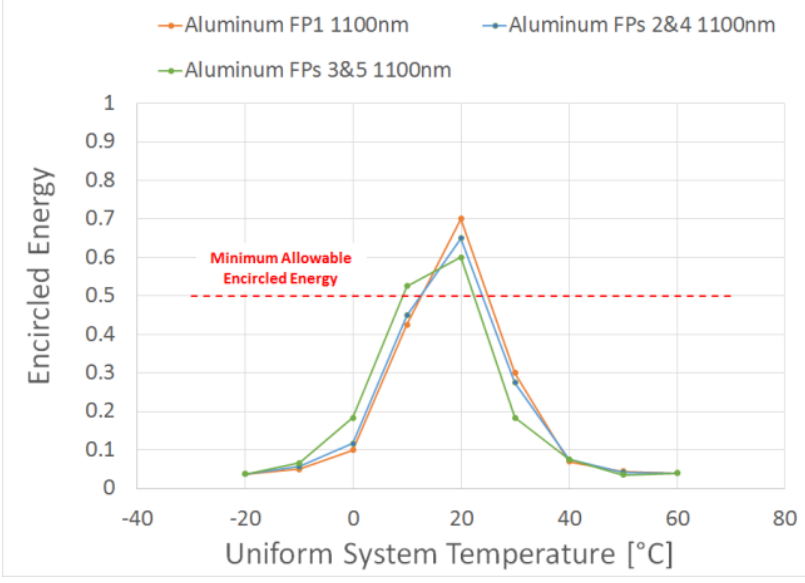

a.) Ritchey-Chrétien, aluminum construction

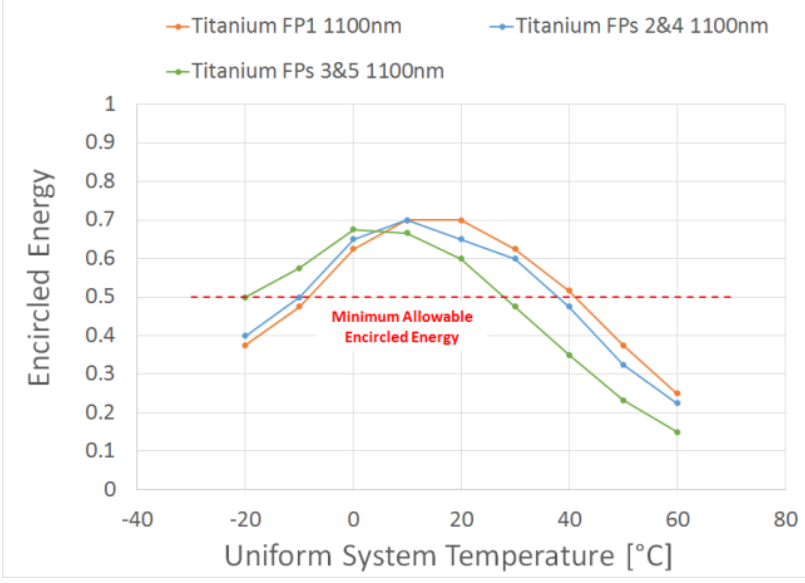

b.) Ritchey-Chrétien, titanium construction

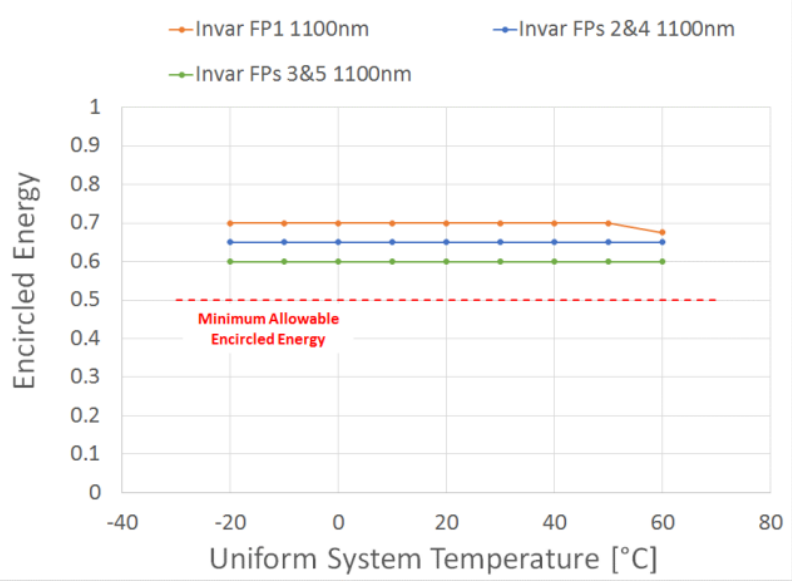

c.) Ritchey-Chrétien, invar construction

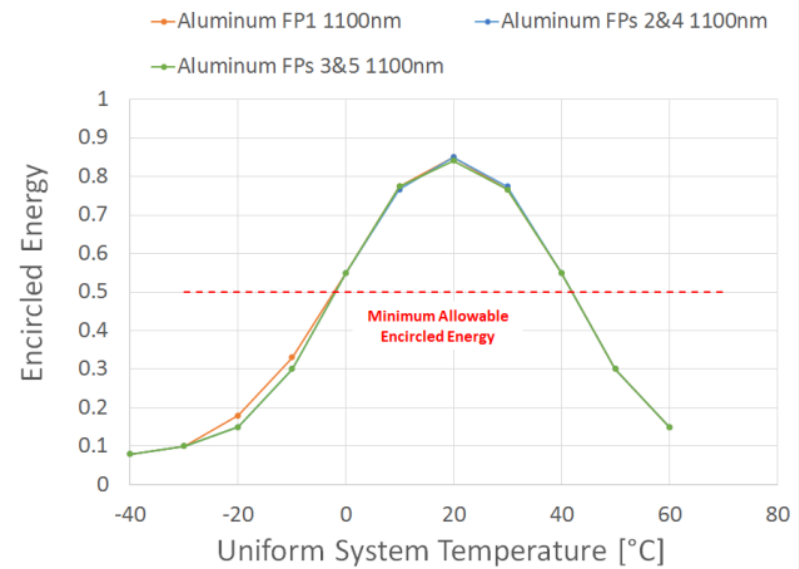

d.) Off-axis, aluminum bench

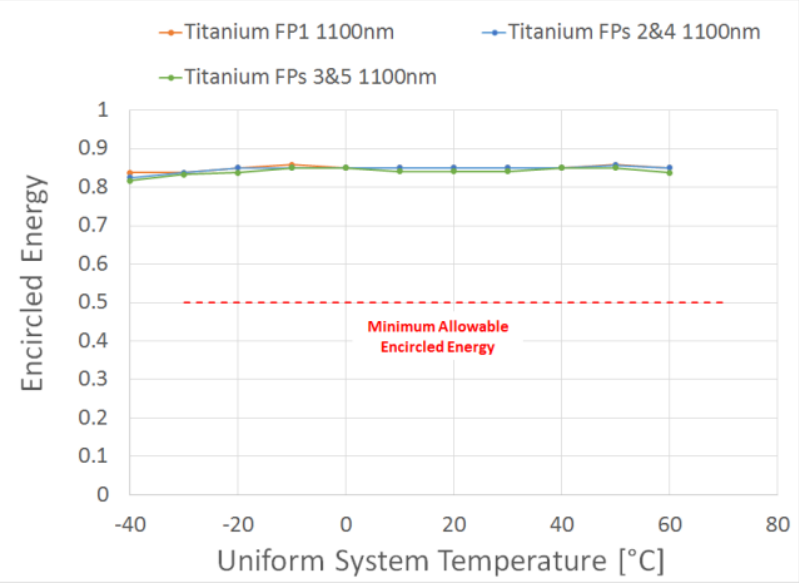

e.) Off-axis, titanium bench

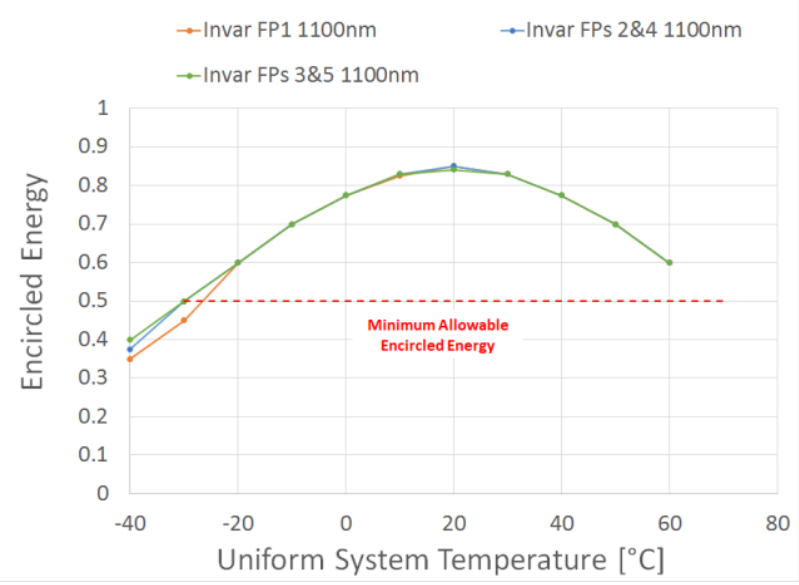

f.) Off-axis, invar bench

Figure 15. Comparison of three bench material choices for each design 


\subsection{Flexure Orientation Results}

An analysis was conducted on telescope mounting orientation with a variety of materials, to assess sensitivity of encircled energy with uniform temperature change. Figure 16 shows the three-point mount locations for each design. Table 7 shows the constraints at each mount for each orientation analyzed. Orientation 1 is fully constrained at all three mount points, while Orientations 2 and 3 assume flexures at two of the mount points. A reference temperature of $20^{\circ} \mathrm{C}$ was used, with analysis temperatures of $\pm 20^{\circ} \mathrm{C}$ from the reference $\left(0^{\circ} \mathrm{C}\right.$ and $\left.40^{\circ} \mathrm{C}\right)$. Results for the two system designs for a single optical bench material are shown in Figure 17 and Figure 18, for 1100nm wavelength. The Ritchey-Chrétien design is fairly insensitive to mounting orientation; even a fully fixed condition. This provides confidence that even a poor flexure design will be sufficient for this system. The off-axis design however is sensitive to mounting orientation, and has very poor performance with a fully fixed design. Care must be taken when implementing an appropriate flexure system. The analyses described in this section, as well as additional cases for various materials were completed in a few hours by two engineers using the template.

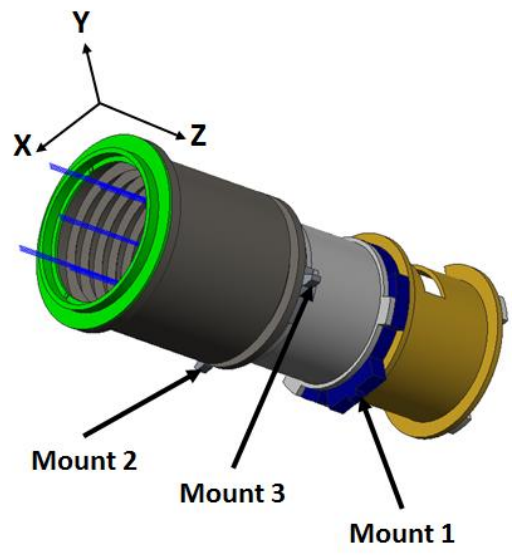

a.) Ritchey-Chrétien mount locations

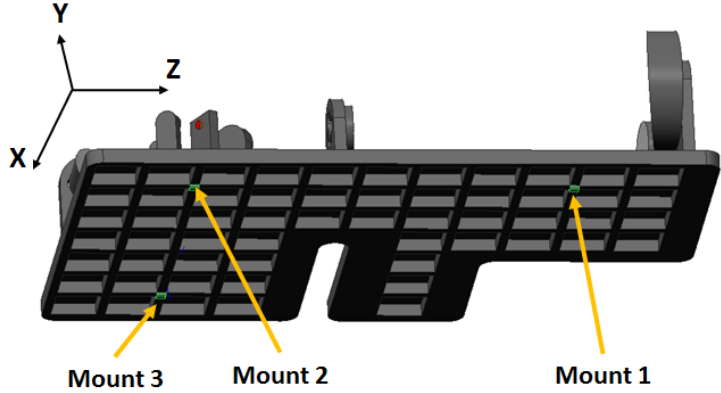

b.) Off-axis mount locations

Figure 16. Mount locations and coordinate system

Table 7. Constraints for each mounting orientation analyzed in both models

\begin{tabular}{|l|c|c|c|l|}
\hline & $\begin{array}{c}\text { Mount 1 } \\
\text { Constraints }\end{array}$ & $\begin{array}{c}\text { Mount 2 } \\
\text { Constraints }\end{array}$ & $\begin{array}{c}\text { Mount 3 } \\
\text { Constraints }\end{array}$ & Description \\
\hline Orientation 1 & XYZ & XYZ & XYZ & Fully constrained \\
\hline Orientation 2 & XYZ & Y & Y & Dual-direction flexures on 2 and 3 \\
\hline Orientation 3 & XYZ & YZ & XY & Single-direction flexures on 2 and 3 \\
\hline
\end{tabular}




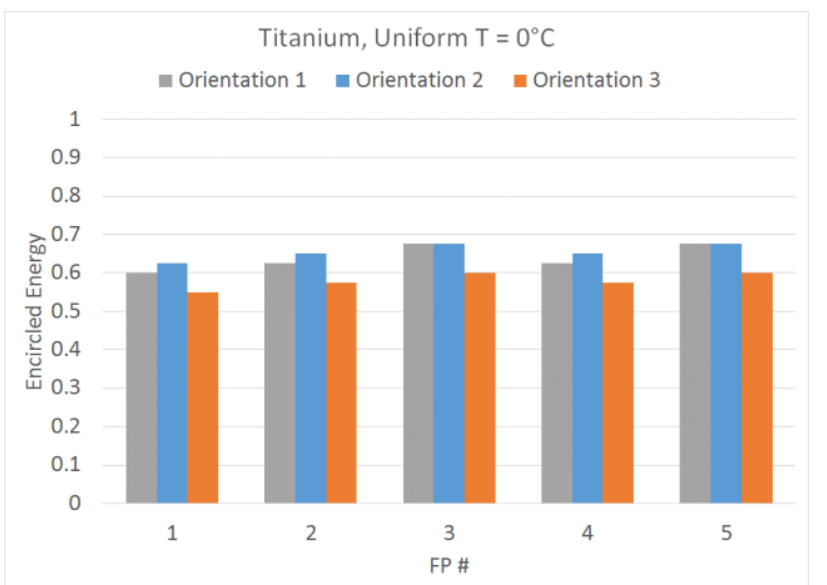

a.) Uniform system temperature $=0^{\circ} \mathrm{C}$

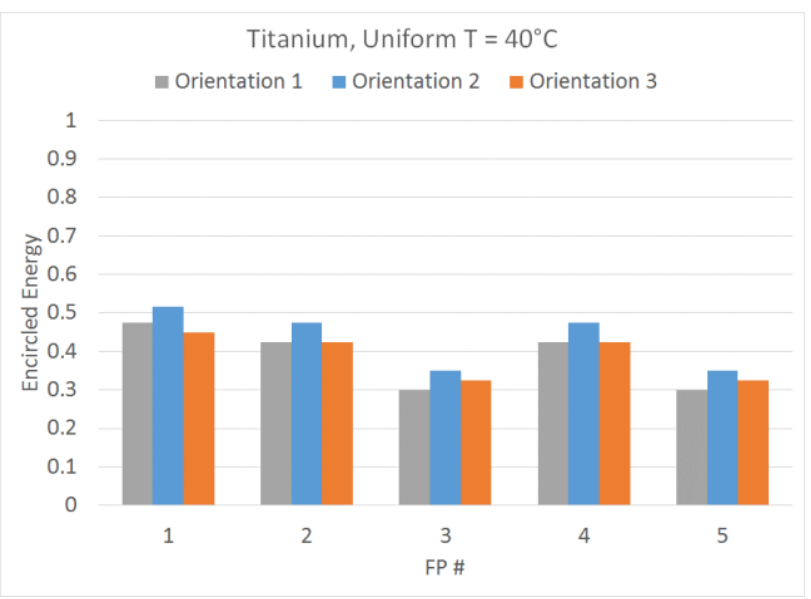

b.) Uniform system temperature $=40^{\circ} \mathrm{C}$

Figure 17. Mounting configuration analysis for Titanium Ritchey-Chrétien design; uniform temperature change of $\pm 20^{\circ} \mathrm{C}$

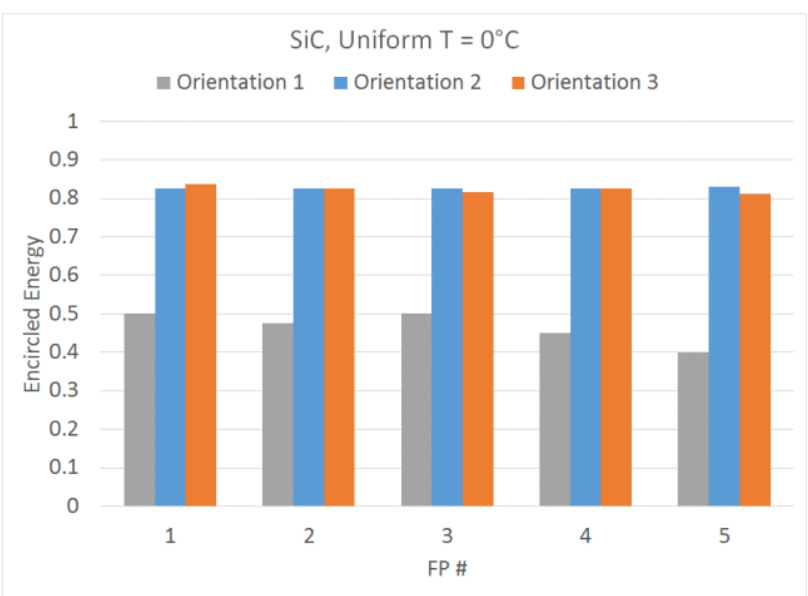

a.) Uniform system temperature $=0^{\circ} \mathrm{C}$

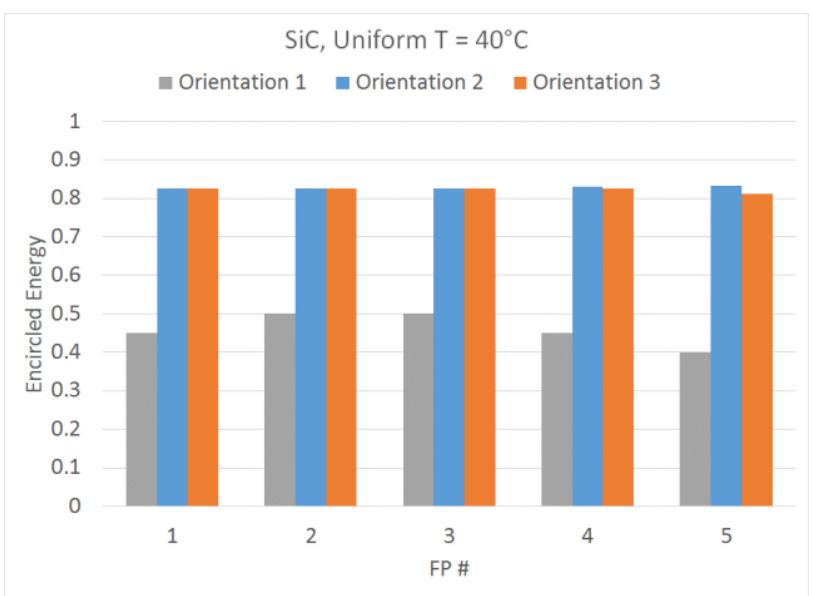

b.) Uniform system temperature $=40^{\circ} \mathrm{C}$

Figure 18. Mounting configuration analysis for Silicon Carbide off-axis design; uniform temperature change of $\pm 20^{\circ} \mathrm{C}$

\subsection{Modal Analysis}

A modal analysis was performed for each design using the modal and harmonic response process in the template. The first and second modes were shown to be insensitive to material choice for the Ritchey-Chrétien design, and met the greater than $50 \mathrm{~Hz}$ requirement, as shown in Figure 19a. These analyses were performed with Orientation 2 in Table 7, which is the least constrained case, and should yield the lowest mode frequencies. For the off-axis design, only the silicon carbide bench met the $50 \mathrm{~Hz}$ requirement for Orientation 2 (Figure 19b). Orientation 3 is required to meet the both the encircled energy requirements, and the modal requirements for this design. Results of this analysis are shown in Figure 20. 


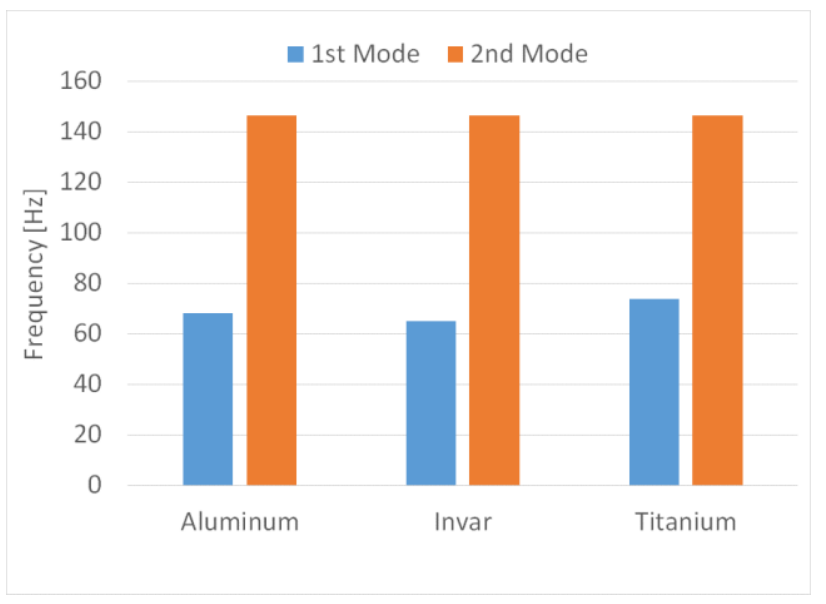

a.) Results for Ritchey-Chrétien design

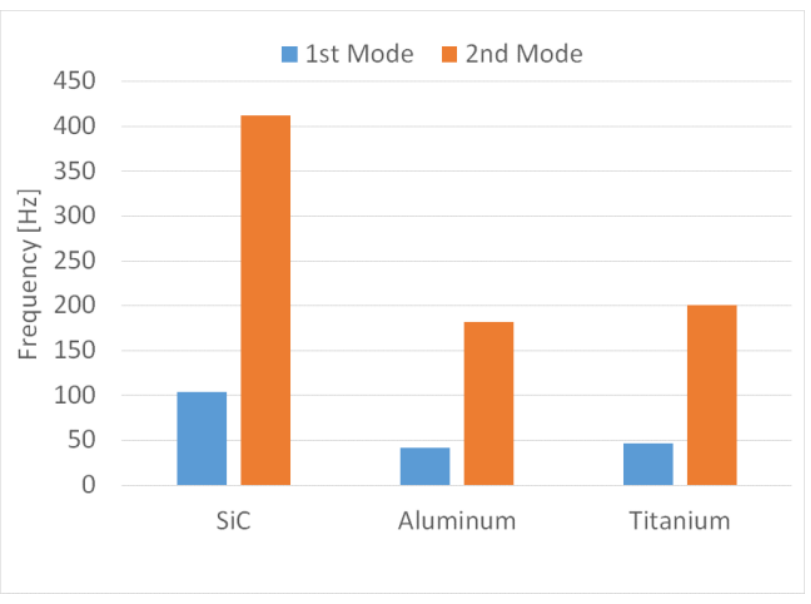

b.) Results for Off-Axis design

Figure 19. Normal mode results for each design with Orientation 2 mounting constraints.

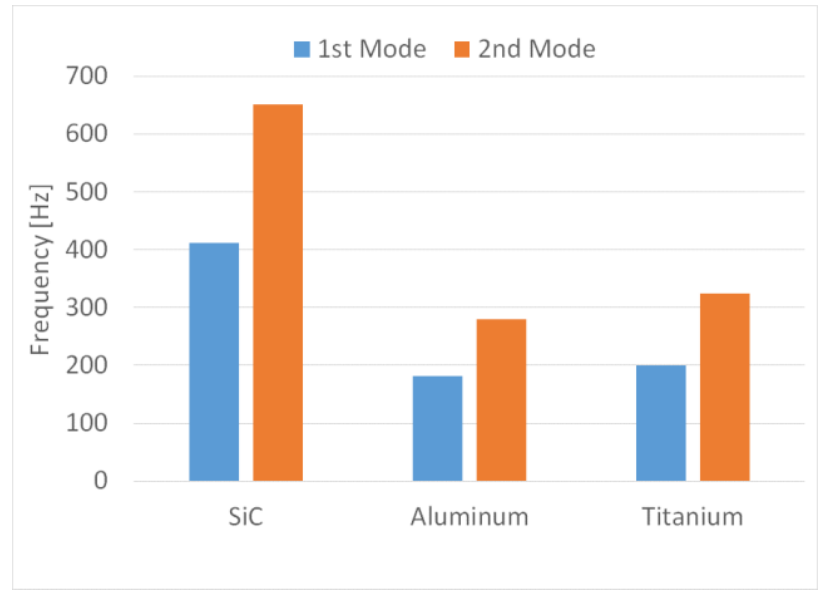

Figure 20. Normal mode results for off-axis design with Orientation 3 mount constraints

\subsection{Orbital Analyses}

Thermal analysis for the on-axis design was performed for operation in low earth orbit similar to the orbit of the International Space Station. Thermal analysis of the off-axis design is forthcoming. To perform the study, the meshed telescope geometry was imported into a Thermal Desktop ${ }^{\mathrm{TM}}$ template file which already contained project-specific geometry for the satellite bus (Figure 21). This process is controlled at the COMET Project Dashboard and specific parameters are controlled from there also, including orbit altitude and beta angle, as well as options for heaters. 


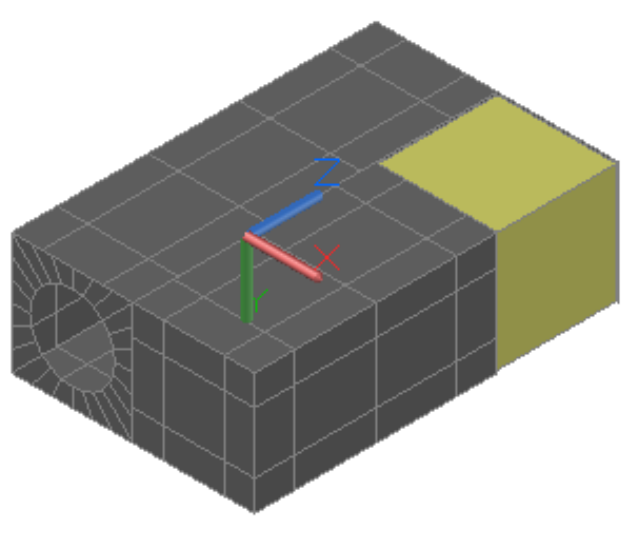

a.) Thermal Desktop ${ }^{\mathrm{TM}}$ template of satellite bus

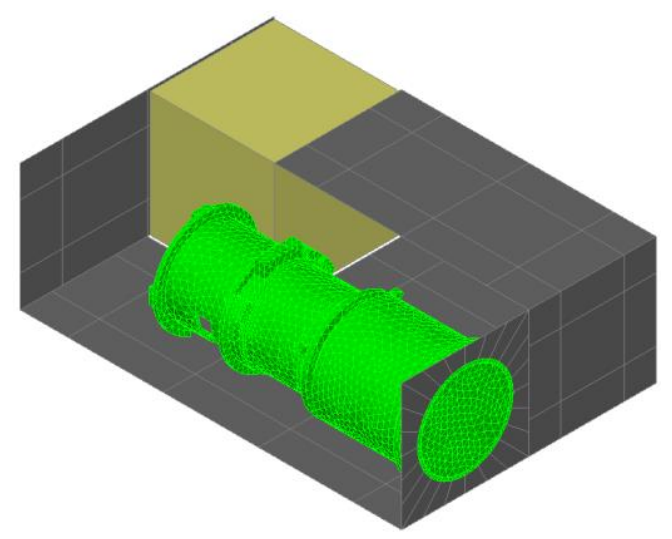

b.) On-axis design imported into template

Figure 21. Thermal Desktop template model for spacecraft bus

Two bounding orbital heating environments were run, a worst-case hot orbit and a worst-case-cold orbit:

Hot Case Orbit: Hot Case Environment Parameters, High Beta Angle (more time in sunlight)

Cold Case Orbit: Cold Case Environment Parameters, Low Beta Angle (more time in shadow)

The thermal response of the on-axis design was studied with an on/off heater applied to the telescope housing (Figure 22) to maintain the telescope at its optimal temperature of $20^{\circ} \mathrm{C}$. The system was also studied with no heater at all. Several material options were explored to make up the telescope housing: aluminum, titanium, and Invar.

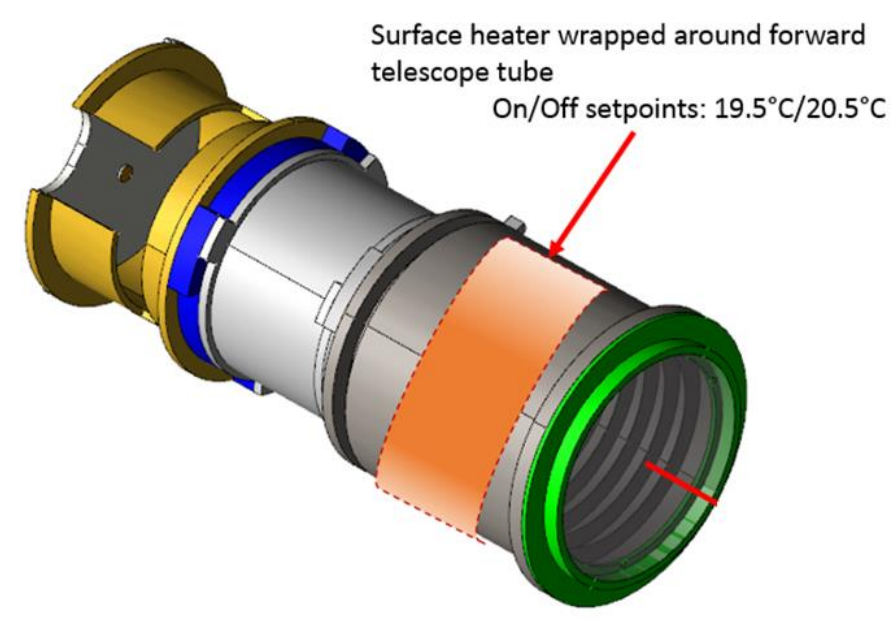

Figure 22. On-Axis Design with Heater Placement Indicated

The temperatures of the satellite housing are dependent on the surface treatment. In this study, all of the satellite's external surfaces are assumed to be aluminum with chromic anodized finish, low a/e. This was used because it resulted in a cold-biased system. The telescope is connected to the satellite at 3 points which makes it very thermally-isolated from the rest of the system. Orbital analyses were run as steady-state averages of the orbit, full transient runs are forthcoming. The hot case temperature results for a titanium telescope are shown in Figure 23.

The first set of analyses were performed without a heater. Figure 24 shows temperature results mapped from the thermal model to the structural model, and the associated deformation predictions for the hot case for both invar and titanium. The temperatures are comparable for the Invar and titanium options because the materials have similar thermal 
conductivity values. The maximum deformation for the Invar design is one order of magnitude smaller than for titanium. Invar has a lower coefficient of thermal expansion than titanium by an order of magnitude. Further comparison showed that the aluminum design had maximum displacements that were an order of magnitude greater than for the titanium option.

Encircled energy result for these cases, as well as an aluminum case are shown in Figure 25. The results expectedly show that the telescope made of Invar was least sensitive to the orbital temperature gradients due to the material's low thermal expansions coefficient. The results for titanium and aluminum are comparable to the uniform temperature change results presented in section 3.2 for $-20^{\circ} \mathrm{C}$, and are slightly better. Without a heater, only the invar case will meet the performance requirement for encircled energy.

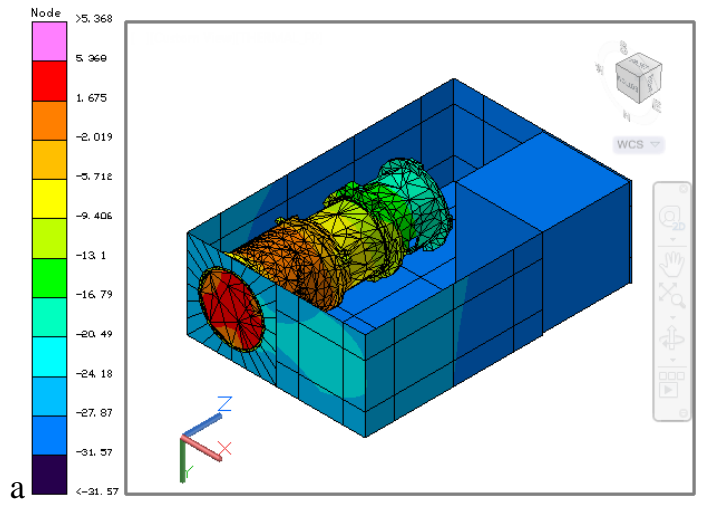

Figure 23. Thermal desktop temperature distribution from hot case titanium, mesh on telescope is coarse to reduce computation time due to large number of radks in radiation ray trace calculations

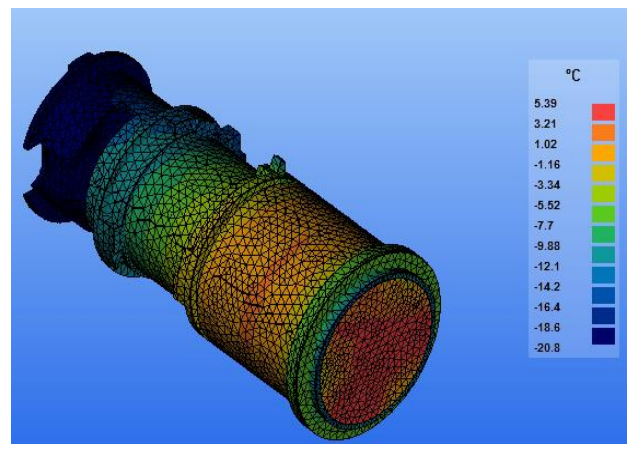

a.) Invar Model, Mapped temperatures

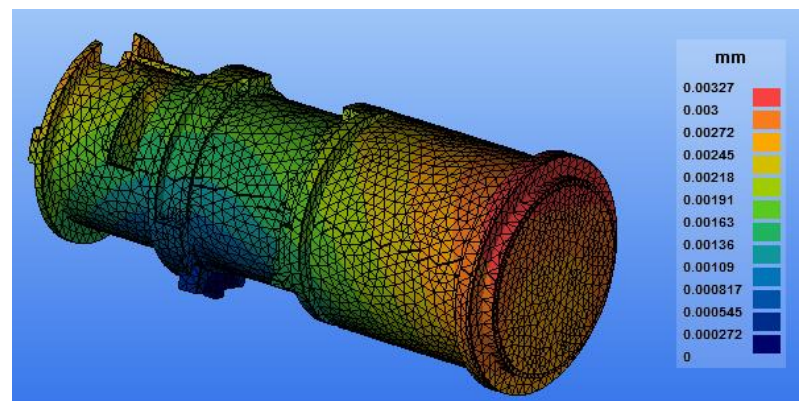

c.) Invar model, predicted deformations

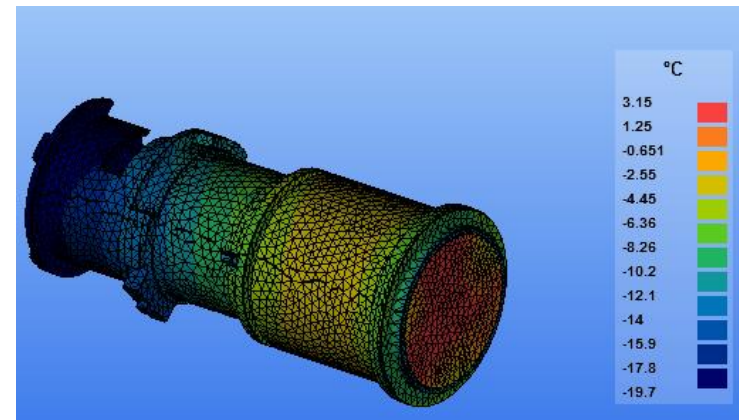

b.) Titanium model, mapped temperatures

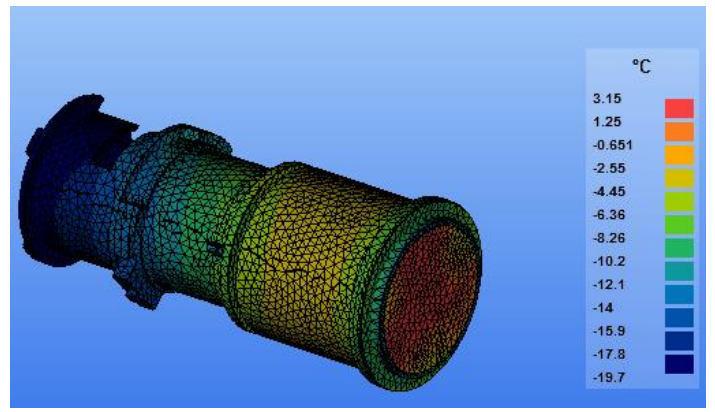

d.) Titanium model, predicted deformations

Figure 24. Hot case temperatures results mapped to structural mesh (a, b). Predicted deformations from NASTRAN (c, d) 


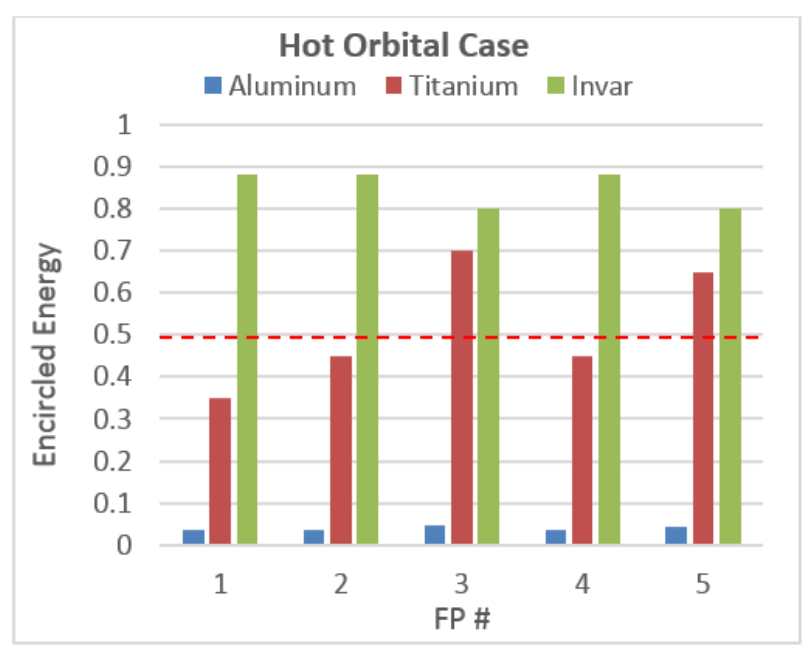

a.) Encircled energy results for hot orbital case

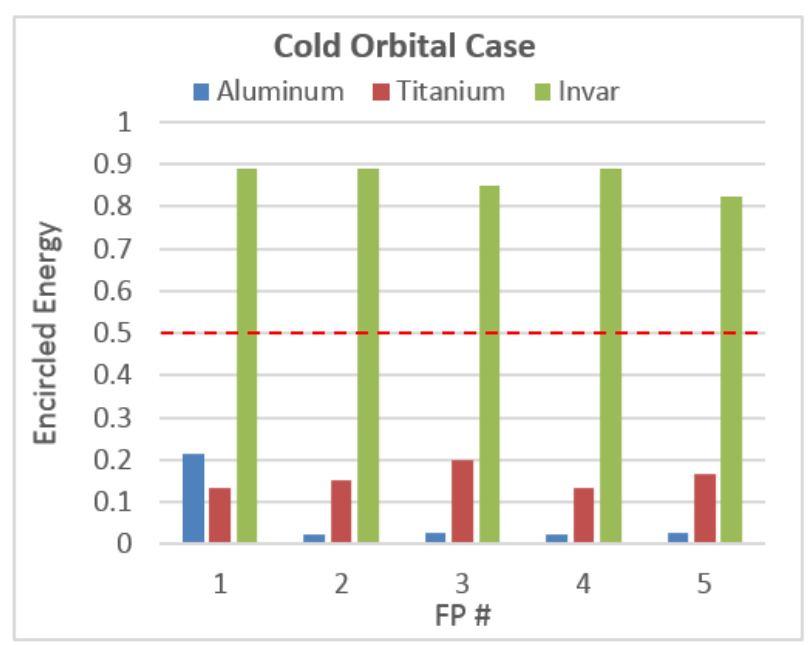

b.) Encircled energy results for cold orbital case

Figure 25. Steady state hot and cold orbital results for Ritchey-Chrétien desgin

The next set of results is for the same designs with the heater on the forward section of the telescope to maintain the optimal alignment temperature for the optical components. Again a steady state orbital analysis is carried out and the necessary heater power required to maintain the heater set point temperature is calculated in each case. The temperature and deformation results are provided in Figure 26. The temperature gradients are less similar than in the unheated case but for both designs the total displacements are reduced, and all material choices meet the encircled energy requirements as shown in Figure 27. The differences arise from the conductivity of the materials and temperature gradient along the un-heated length of the telescope tube.

Figure 28 shows the average power required by the heater over the course of an orbit. Titanium outperforms Invar in this case by requiring the least power. The result is not surprising since titanium has lower thermal conductivity than either Invar or aluminum. Further analysis can be used to optimize the design, by adding additional heaters along the length, and adding multi-layer insulation to reduce the required heater power. The STOP template makes these types of optimization studies easy to perform, and can be performed by the thermal engineer alone.

An obvious tradeoff exists between the mass requirements and power requirements for the project. Using a titanium telescope would have lower power needs, but would weigh over 50\% more than an aluminum telescope. Using Invar for the telescope would mean that no heaters would be necessary, but would weigh approximately $200 \%$ more than an aluminum telescope of the same dimensions. The ability to quickly run trade studies using the template provides more data for engineers to make decisions. 


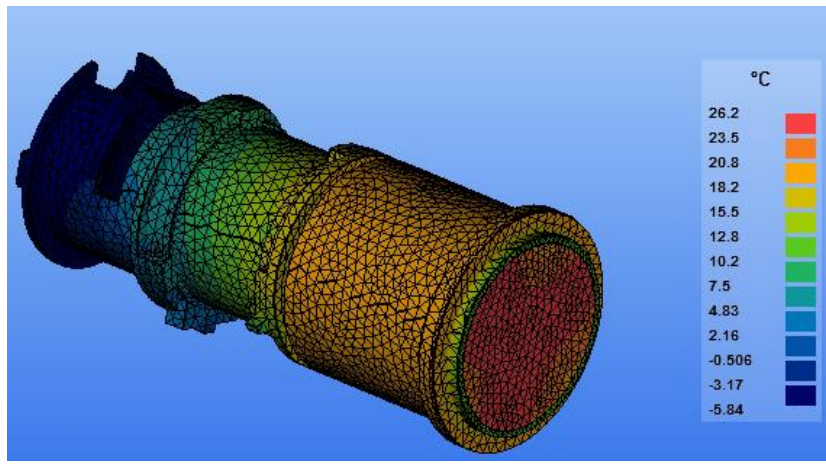

a.) Invar Model, Mapped temperatures

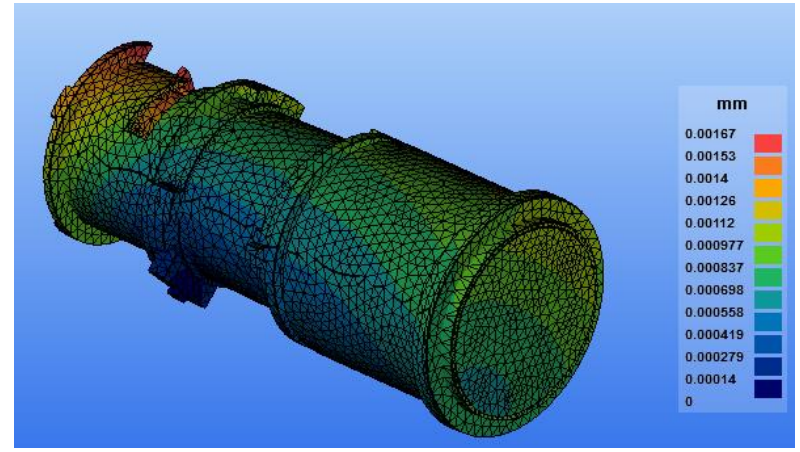

c.) Invar model, predicted deformations

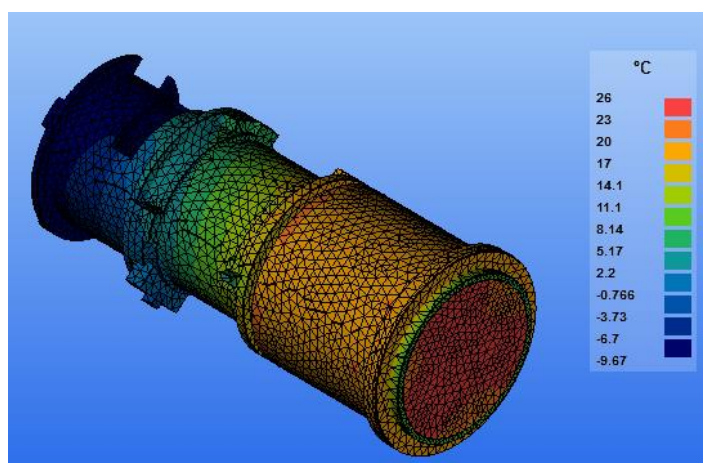

b.) Titanium model, mapped temperatures

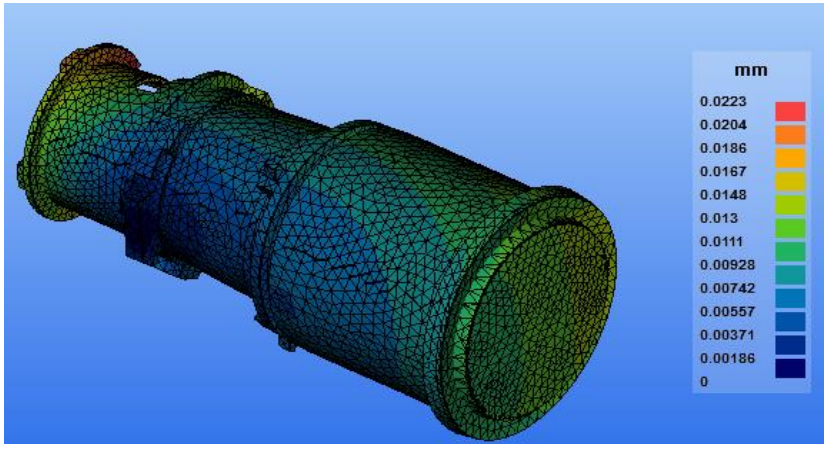

d.) Titanium model, predicted deformations

Figure 26. Hot case temperatures results with included heater mapped to structural mesh (a, b). Predicted deformations from NASTRAN (c, d)

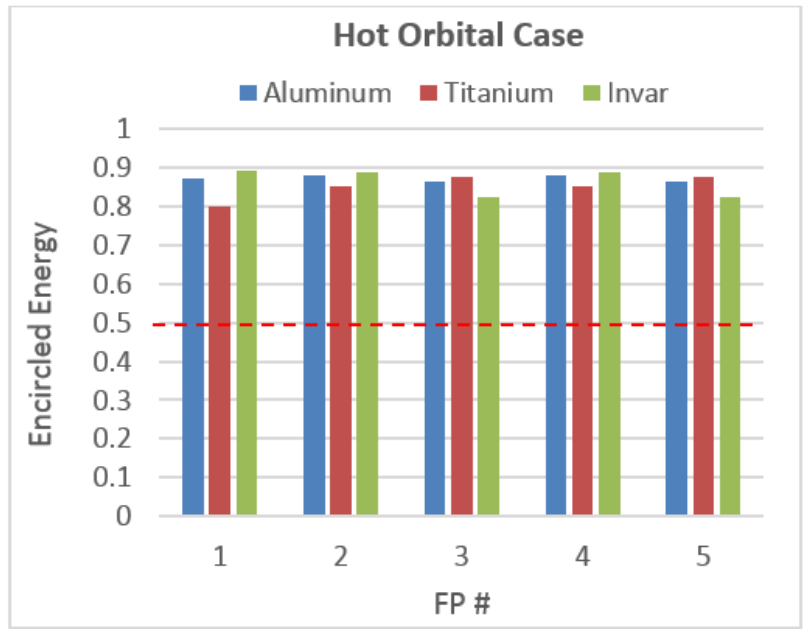

a.) Encircled energy results for hot orbital case

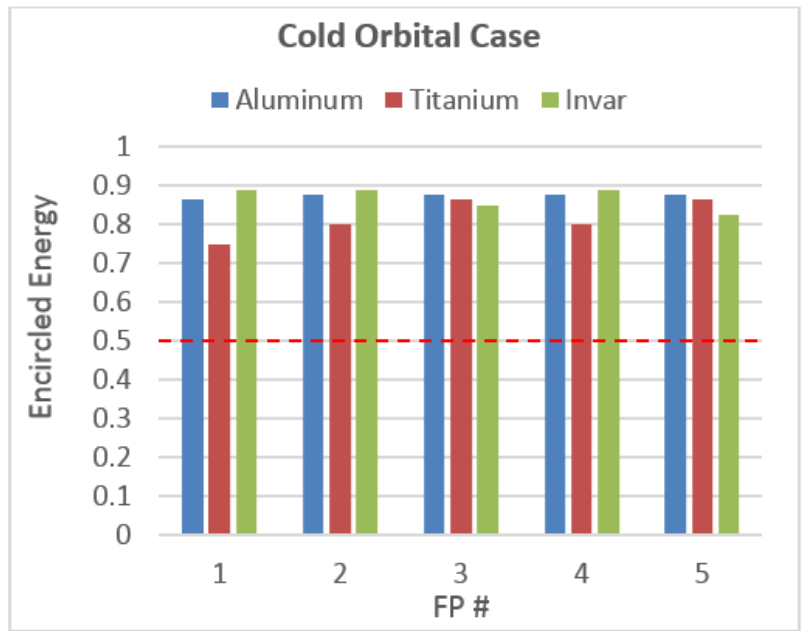

b.) Encircled energy results for cold orbital case

Figure 27. Steady state hot and cold orbital results for Ritchey-Chrétien design, including a heater 


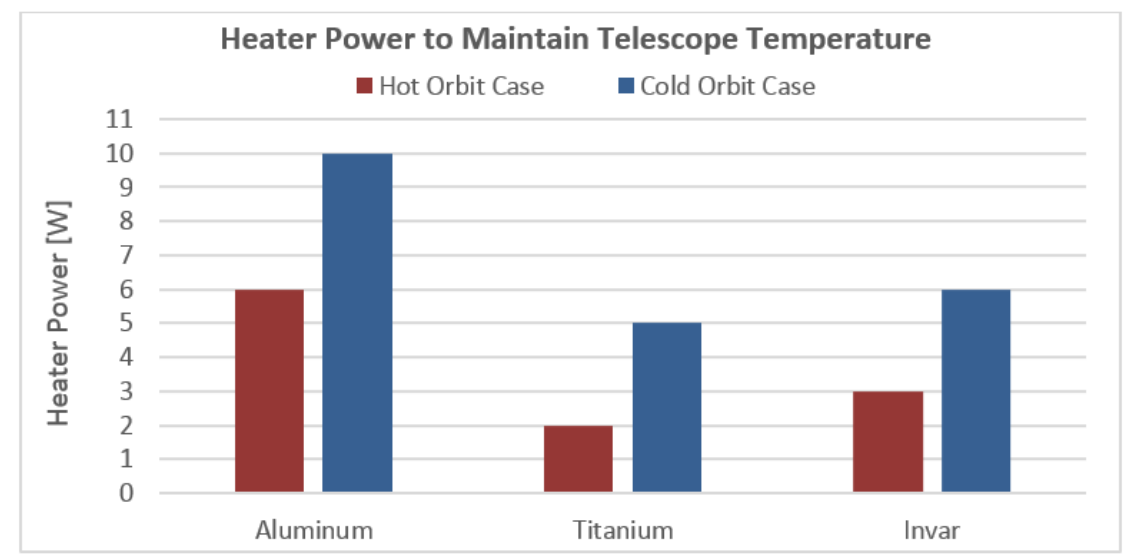

Figure 28. Steady state heater power requirements for each orbital case

\section{SUMMARY}

The generic STOP template enables engineers to test more potential design solutions in a shorter amount of time than is possible with the traditional method. The template relies on the fact that optical systems are functionally similar, and contain the same type of hardware, such as optical elements, mechanical mounts, and optical benches. This fact enables the definition of a standard naming convention that can be used to generically define analysis processes and outputs that can be used to verify a set of requirements. This can be done before the generation of a CAD or optics model, which lets any optical system use the template, and eliminates duplicated upfront work across projects. This allows the engineering and project team to spend more time defining the analyses, and design options that should be studied, and less time performing the more tedious tasks of setting up models, and transferring data. The fact that the model is self-contained is also a significant benefit, and allows a working STOP model to be saved, and used later in the project for anomaly investigation, or on-orbit performance correlation. Based on the work performed on the two optical systems described, it is estimated that $50 \%$ to $75 \%$ of the time spent performing a particular analysis cycle can be reduced. These savings are attributed partly to the automation of the analysis flow, and partly to the ability of each engineer on the team to run a full STOP analysis independently. The latter ability is particularly useful since it allows the team to perform all trade studies in parallel rather than sequentially. Work is planned to expand the template to include additional analyses, and requirements verification in the coming year.

\section{REFERENCES}

[1] Bolognese, J. and Irish, S., "Structural-Thermal-Optical-Performance (STOP) Analysis," TFAWS (2015). http://ntrs.nasa.gov/archive/nasa/casi.ntrs.nasa.gov/20150017758.pdf

[2] Comet Solutions®, http://cometsolutions.com/

[3] Geis, J., Et. Al., "Collaborative design and analysis of Electro-Optical sensors," Proc. SPIE 7427, 74270H-1 (2009).

[4] Scola, S., Et. Al., " Structural-Thermal-Optical-Performance (STOP) Model Development and Analysis of a Fieldwidened Michelson Interferometer," Proc. SPIE 9193, 91930I-2 (2014). 